\title{
Sobre los determinantes de la colusión en las compras públicas: el caso de Chile
}

\section{The determinants of collusion in public procurement: the case of Chile}

\author{
JUAN FRANCISCO MARTÍNEZ S., DAVID ESCOBAR Y \\ CLAUDIO LOYOLA*
}

Resumen: Este artículo intenta explicar los determinantes de la colusión y sus consecuencias económicas para las compras públicas. Empleando una amplia variedad de modelos econométricos para la colusión, el documento concluye que la colusión es generada principalmente por el sector productivo, la independencia del organismo comprador, el monto de dinero involucrado, la posibilidad de subcontratación, la cantidad de ofertas y el esquema de adjudicación múltiple. Después de esta investigación, se implementó un enfoque econométrico estándar de regresiones MCO, Probit y Logit para obtener los determinantes, su significancia y su magnitud. Las variables independientes y dependientes fueron escogidas a partir de un profundo análisis bibliográfico, junto con consultas a los expertos en el organismo chileno de compras públicas. Los resultados de este estudio no son completamente concluyentes, debido a que estamos considerando posibilidades de colusión, sin el correspondiente juicio legal.

Palabras clave: Compras públicas -colusión - corrupción y competencia.

Abstract: This article tries to explain the determinants of collusion and its economic consequences for public procurement. Employing a wide variety of models econometric collusion, this paper concludes that collusion is generated mainly by the productive sector, the independence of the buyer, amount of money involved, the possibility of outsourcing, the number of bids and awarding scheme. After this research, a standard econometric approach to OLS was implemented, Probit and Logit for determinants, their significance and magnitude. The independent and dependent variables were chosen following a thorough literature review, along with consultation with experts in the Chilean procurement agency. The results of this study are not fully conclusive, because it is considering possibilities of collusion, without proper legal trial.

Key words: Procurement - collusion - corruption - competence

* Los autores pertenecen a la división de Mercado Público del departamento de Investigación, Dirección de Compras y Contratación Públicas de Chile Compra, como parte del Ministerio de Hacienda de Chile. 


\section{INTRODUCCIÓN}

La Dirección de Contratación y Compras Públicas es la institución responsable de proveer una exclusiva y centralizada plataforma para ejecutar todas las compras que requieren los organismos públicos en Chile. En este sentido, uno de los principales objetivos de esta institución es generar las condiciones para un mercado eficiente y no discriminatorio. Así, esta entidad busca proporcionar los medios para lograr la transparencia, y por lo tanto es fundamental preguntarse cómo lograr la libre competencia y el comportamiento no-coludido.

Según su experiencia en el trabajo cotidiano, nuestros funcionarios saben que la colusión puede afectar a los procesos de dos maneras principales. Por un lado, la colusión no permite la libre competencia o intercambio necesarios para obtener precios eficientes; es decir, ella genera graves pérdidas económicas a través de pagos excesivos o injustificados —especialmente en el caso de la colusión entre proveedores-. Por otro lado, daña seriamente la percepción de transparencia —especialmente en el caso de la colusión entre un proveedor y un comprador público-.

La colusión se previene y detecta utilizando algunas herramientas principales. En primer lugar, como una herramienta de prevención, los organismos públicos compradores pueden utilizar checklists, o listas de control $^{1}$, para preparar sus bases de licitación y contratos. Una segunda herramienta valiosa es el checklist de detección, que se utiliza una vez abierto el proceso de licitación, para percibir cualquier irregularidad que afecte negativamente una competencia normal. Un tercer método en la lucha contra la colusión es detectar patrones de comportamiento utilizando un procedimiento de data mining, o minería de datos. Aunque este procedimiento no es concluyente, ofrece una perspectiva valiosa acerca de cuáles son los sectores más susceptibles a la colusión, lo cual permite al supervisor utilizar recursos limitados de manera eficiente.

Finalmente, este estudio proporciona un marco inicial para detectar la manifestación de eventos de colusión. El valor agregado de esta investigación está basado en el uso y en el procesamiento de datos crudos, mediante la aplicación de pruebas econométricas estándares.

\section{HECHOS ESTILIZADOS DE COLUSIÓN}

La colusión en las licitaciones públicas se produce en dos dimensiones. La primera es cuando las empresas se ponen de acuerdo para aumentar sus ganancias de manera ilícita. En las licitaciones públicas, estas se ponen de acuerdo para aumentar sus ofertas para obtener beneficios adicionales al proporcionar el bien o servicio requerido. En este caso, los

1 Es un instrumento educacional concebido para clarificar la legislación y los procedimientos de compras públicas. 
proveedores utilizan mecanismos múltiples y capaces de evolucionar, lo cual dificulta bastante su regulación. Existen varios ejemplos célebres en la literatura ${ }^{2}$, con pérdidas millonarias para los gobiernos.

El segundo tipo de colusión es el ilícito, en el cual el comprador acuerda compartir con el proveedor las ganancias extraordinarias producidas por un aumento ilegal del precio adjudicado. A menudo este fenómeno recibe el nombre de "corrupción" en vez de "colusión"; sin embargo, lo único que cambia son los agentes involucrados en el acuerdo ilícito, y la magnitud de las pérdidas — que son menores-. Aunque las pérdidas económicas causadas por este fenómeno son menores, este sufre de graves daños políticos y daños económicos indirectos, ya que genera una percepción de falta de transparencia del sistema, lo cual lleva a los proveedores a disminuir su participación. Ello limita la competencia y genera precios ineficientes.

La colusión es una grave distorsión para cualquier sistema de compras públicas porque no permite que la competencia conduzca a precios eficientes. Mientras mayores sean los precios, más gastos tendrá el gobierno. La competencia en este mercado genera un ahorro con respecto a situaciones en las cuales los agentes no deben competir directamente, como los procesos de compras estándares de una unidad aislada sin poder de compra. Las asociaciones ilícitas obtienen recursos adicionales del gobierno, lo cual aumenta el gasto público; también contribuyen a la inflación y destruyen la confianza en la economía, y así socavan completamente el sistema de la economía de mercado.

\section{I.1. Descripción del mercado y algunas reglas generales}

Durante el año 2007, se emitieron más de 1,5 millones de órdenes de compra, por un monto total de US\$ 4458 millones. La cantidad de licitaciones públicas fue de 450 023. Las siguientes tablas muestran en detalle algunos valores significativos y describen parcialmente la competitividad del mercado de compras públicas en Chile.

SOBRE LOS

DETERMINANTES DE LA COLUSIÓN EN LAS COMPRAS PÚBLICAS: EL CASO DE CHILE

THE DETERMINANTS OF COLLUSION

IN PUBLIC PROCUREMENT: THE CASE OF CHILE 
Tabla 1

\begin{tabular}{lrrrrr}
\hline \multicolumn{1}{c}{ Región } & Micro & Pequeñas & Medianas & Grandes & Total \\
\hline Arica y Parinacota & 7462 & 9188 & 6029 & 15223 & 37902 \\
Tarapacá & 19359 & 19729 & 11035 & 29428 & 79550 \\
Antofagasta & 2106 & 3091 & 1856 & 6222 & 13275 \\
Atacama & 4625 & 5091 & 4450 & 11629 & 25796 \\
Coquimbo & 3463 & 4690 & 2304 & 5943 & 16401 \\
Valparaíso & 35853 & 42665 & 23003 & 60590 & 162111 \\
Metropolitana & 8690 & 9429 & 7304 & 15363 & 40786 \\
Lib. Gral. Bdo. O'higgins & 10576 & 12322 & 5488 & 16825 & 45211 \\
Maule & 17733 & 20456 & 9527 & 25137 & 72853 \\
Bío-Bío & 9588 & 9878 & 4248 & 17338 & 41052 \\
Araucanía & 6415 & 6606 & 4523 & 12658 & 30202 \\
Los Ríos & 17324 & 17178 & 8909 & 27377 & 70787 \\
Los Lagos & 114424 & 148274 & 96789 & 312100 & 671587 \\
Aysén & 3927 & 3397 & 1903 & 7437 & 16663 \\
Magallanes y Antártica & 28204 & 38054 & 25071 & 75551 & 166880 \\
Total & 289747 & 350047 & 212441 & 638820 & 1491054 \\
\hline
\end{tabular}

Elaboración propia.

Tabla 2

\begin{tabular}{lrrr}
\hline Maule & 28732 & 4194 & $14,60 \%$ \\
Bío-Bío & 61745 & 8505 & $13,80 \%$ \\
Araucanía & 28181 & 3900 & $13,80 \%$ \\
Los Ríos & 12845 & 1516 & $11,80 \%$ \\
Los Lagos & 27179 & 3621 & $13,30 \%$ \\
Aysén & 8459 & 1265 & $14,90 \%$ \\
Magallanes y Antártica & 14467 & 2182 & $15,10 \%$ \\
Total & 450023 & 65172 & $14,50 \%$ \\
\hline
\end{tabular}

Elaboración propia.

Tabla 3

\begin{tabular}{lc}
\hline \multicolumn{1}{c}{ Región } & $\%<3$ Ofertas \\
\hline Arica y Parinacota & $40,60 \%$ \\
Tarapacá & $39,62 \%$ \\
Antofagasta & $41,25 \%$ \\
Atacama & $37,93 \%$ \\
Coquimbo & $39,90 \%$ \\
Valparaíso & $31,17 \%$ \\
Metropolitana & $30,18 \%$ \\
Lib. Gral. Bdo. O’higgins & $30,67 \%$ \\
Maule & $31,04 \%$ \\
Bío-Bío & $30,60 \%$ \\
Araucanía & $34,68 \%$ \\
Los Ríos & $31,01 \%$ \\
Los Lagos & $37,14 \%$ \\
Aysén & $48,54 \%$ \\
Magallanes y Antártica & $35,28 \%$ \\
Total & $32,67 \%$ \\
\hline
\end{tabular}




\subsection{Marco teórico de la colusión}

En esta sección describiremos brevemente la teoría económica básica del fenómeno de la colusión y ofreceremos algunas explicaciones racionales para la parte empírica de este estudio. Ya sabemos que los procesos que estamos modelando se definen como licitaciones (subastas públicas). Por lo tanto, las herramientas que vamos a utilizar están relacionadas con la teoría de las subastas y los juegos.

Si se aplica un análisis estándar de la teoría de juegos, un acuerdo de colusión es un equilibrio subperfecto en el caso de los juegos de una sola vez (one-shot game), pero no está claramente definido en el caso de los juegos repetidos —el caso más realista—. Sin embargo, puede ser un equilibrio Nash en cualquier especificación de juego, dependiendo de los supuestos. En este sentido, el juego debería resolverse de manera dinámica.

La solución de este problema es bastante intuitiva: las ganancias de la colusión deberían ser mayores que el castigo en caso de una "traición" al acuerdo. A partir de este análisis, es fácil deducir que, mientras mayor sea la cantidad de ofertas, mejor será el precio que puede obtener un organismo comprador centralizado, ya que, a medida que aumenta la cantidad de ofertas - $\mathrm{u}$ oferentes en este caso-, los incentivos para coludirse se reducen. Sin embargo, los supuestos de este análisis son muy fuertes y no implican una ausencia de colusión en casos reales, aunque proporcionan la principal evidencia teórica de nuestro estudio.

Por otro lado, la colusión es un equilibrio muy difícil de predecir ${ }^{3}$ y aún más difícil de detectar; es más, en ciertos casos es dinámica, mientras que en otras oportunidades es muy estable. Lo único que se puede lograr observando los datos es detectar posibilidades de colusión ${ }^{4}$, basados en patrones anormales de comportamiento entre los oferentes y las ofertas. Aun así, este procedimiento no asegura realmente que exista colusión en alguna transacción en particular. Para que exista colusión un juez debe revisar la ley y determinarlo basado en pruebas y evidencias.

Dadas las ideas de esta sección, construiremos un conjunto de variables que representan posibilidades de equilibrios a través de la colusión, basadas en un comportamiento anormal de los oferentes y las ofertas, donde exista una coincidencia entre la teoría y la práctica. Es decir, analizaremos eventos con componentes no-competitivos, con precios anormalmente altos o precios falseados (dummy prices), ganadores repetidos, $\mathrm{u}$ oferentes nuevos extremadamente afortunados. 
El contexto teórico de este documento es una competencia "oligopolística" para el caso de la colusión entre proveedores $(\mathrm{O}-\mathrm{O})$ y de comportamientos no-competitivos en el caso de la colusión entre un proveedor y un comprador (O-D). En ambos casos existe una ganancia anormal, no-competitiva e ilícita, que se comparte entre los jugadores, que es inversamente proporcional a la cantidad de jugadores ${ }^{5}$.

La cifra de la ganancia ilegal se puede determinar a partir de los datos agregados al comparar los precios estimados con la cifra adjudicada; sin embargo, esto implicaría una labor titánica, si consideramos que la diferencia contiene elementos no necesariamente relacionados con la colusión. Aun así, se ha demostrado que los efectos sobre las licitaciones $(\mathrm{O}-\mathrm{O})$ analizadas son enormes ${ }^{6}$ en términos de cifras monetarias. Por otro lado, los efectos sobre las licitaciones (O-D) analizadas son menores, pero no menos importantes, ya que la imagen de transparencia y credibilidad del sistema está vinculada a estas irregularidades. A mayores irregularidades, menor percepción de transparencia; por lo tanto, dado que la transparencia aumenta la intención de participar, constituye un componente muy importante para la competencia y la obtención de precios eficientes.

Así, en ambos casos de colusión, para calcular el impacto de los valores intrínsecos de las licitaciones sobre la colusión, lo primero que se debe hacer es establecer cuáles son las condiciones que facilitan la colusión? Una vez que tenemos las variables proximales, ponemos a prueba las condiciones y obtenemos la significancia de cada determinante en la dimensión de la colusión que estamos analizando.

\section{I.3. Proxies de colusión}

Según describimos anteriormente, modelaremos la colusión con proxies relacionados a la posibilidad de colusión. De acuerdo a la literatura revisada (Porter y Zona 1992, Hendricks y Porter 1989), algunas de las anomalías detectables más comunes son adjudicación en la primera oferta, ganador repetido, demasiadas ofertas falsas y precios adjudicados excesivamente altos.

El primero de estos conceptos es bastante claro: es un hecho muy singular que un proveedor nuevo gane la primera licitación en la que participa; existen serias dudas sobre el proceso, aunque es solo una posibilidad de colusión - fuerte, pero de todas formas solo una posibilidad-.

\footnotetext{
5 Shapiro, C. "Theories of oligopoly behavior". En R Schmalensee y R. Willig (editores). Handbook of industrial procurement. Ámsterdam: North Holland.

6 Klemperer, Paul. Bidding markets. UK, 2005.

7 Hendricks, K. y R. Porter. "Collusion in auctions". Annales d'Economie et de Statistique, 1989.
} 
El segundo concepto es directo: es extraño que el mismo proveedor sea adjudicado periódicamente. Definitivamente podría existir colusión entre el demandante y el proveedor, aunque esto no necesariamente implica "malas intenciones"; simplemente podría significar que un organismo comprador favoreció a un proveedor "favorito" porque puede confiar en este sin revisar la información sobre la competencia.

La tercera manifestación es más sutil que las anteriores, aunque no menos importante. En un proceso con muchas ofertas que se acercan al precio adjudicado, la posibilidad de que los proveedores hayan llegado a un acuerdo para imponer un precio caro y repartirse la torta aumenta dramáticamente.

El cuarto síntoma de la colusión es muy directo pero difícil de detectar. Utiliza el precio estimado que establece el comprador y lo compara con el precio adjudicado. Si la diferencia es anormalmente grande, es evidente que existe colusión (O-O o O-D). Sin embargo, no podemos excluir los procesos en que se realizaron evaluaciones imprecisas a causa de una mala gestión, y no necesariamente con malas intenciones.

\subsection{Determinantes de la colusión}

De acuerdo con la literatura sobre la evidencia cualitativa y algunas evidencias cuantitativas de colusión en las compras públicas, podemos describir un conjunto de determinantes de la colusión. Es decir, algunas variables sensibles pueden afectar la posibilidad de colusión en los procesos de licitaciones públicas.

Hemos construido una base de datos que contiene toda la información significativa para detectar la colusión en licitaciones públicas. En nuestro modelo, el dato atómico es la licitación, que tiene ciertos atributos que se describen a continuación.

Utilizamos la posibilidad de subcontratación de una licitación como una variable ficticia, dado que este grado de libertad puede afectar los incentivos para la libre competencia. Una segunda variable es que la fecha de publicación (registros) nos permite controlar por tendencias de tiempo y también nos da una idea acerca de si la colusión está aumentando o disminuyendo con el paso del tiempo. Una tercera variable es la cantidad de ofertas, utilizada para observar en qué sentido la competencia afecta las posibilidades de colusión. Las variables cuarta y quinta son la cantidad de proveedores adjudicados y la categoría de adjudicaciones múltiples, para controlar por el efecto combinado sobre la competencia de la licitación. Una sexta variable es el monto total adjudicado, que controla por los incentivos tomando en cuenta el tamaño de la torta. Una séptima variable es el tamaño de la empresa, en términos de su cantidad de ventas; esto determinaría cuál sector de empresas tiene mayores posibilidades 
de colusión. Una octava variable a considerar es la cantidad de días de decisión desde la publicación de una licitación hasta su adjudicación, para ver si un mayor período de tiempo genera un entorno más competitivo. También utilizamos una novena variable del exceso de ofertas, que es igual a "1" si la cantidad de ofertas es mayor a ocho; esto es para controlar por situaciones no-lineales en el proceso competitivo. Una décima variable que utilizamos es la cantidad de unidades de compras del organismo comprador, y el razonamiento en este caso es que, a mayor cantidad de unidades de compras, menos independientes son las unidades de compras dedicadas a generar la licitación, ya que deben consultar o responder ante un organismo centralizado. El undécimo conjunto de variables son las variables ficticias geográficas regionales, para controlar por concentración de compras regionales. Un duodécimo conjunto de variables es el tamaño de la licitación, en términos del monto de dinero involucrado; para controlar por el tamaño del incentivo. Finalmente, utilizamos la clasificación del producto o servicio de los códigos del nivel uno de la ONU para determinar qué áreas de negocios afectan más las posibilidades de colusión, debido a características intrínsecas de los mercados.

\section{Gráfico: Component loadings}

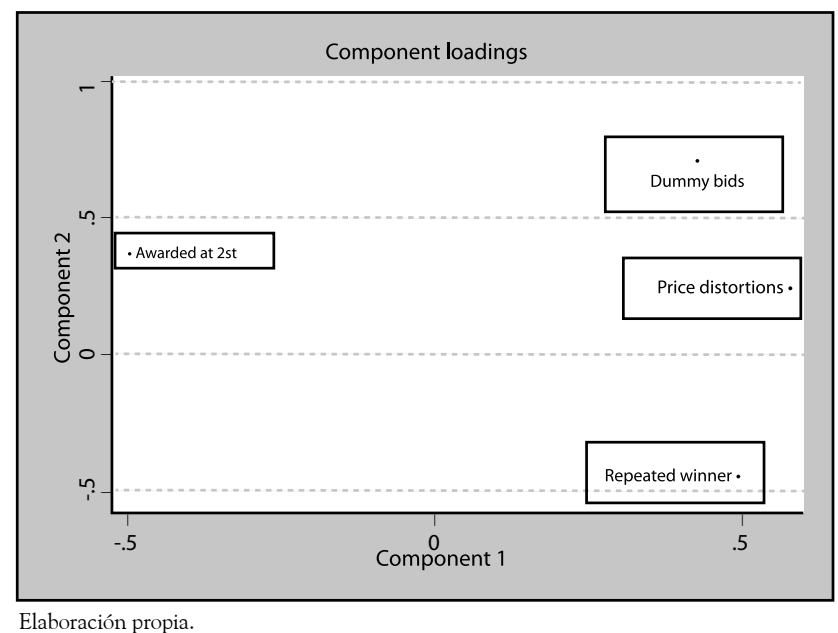

\section{EVIDENCIA EMPÍRICA}

La metodología de esta investigación consiste en regresiones de corte transversal $\mathrm{MCO}$ y un análisis de regresión binaria probabilística. Esta metodología permitirá explicar los efectos causales relacionados con la colusión. Buscaremos los determinantes de la posible colusión y describiremos las explicaciones de los patrones de comportamiento. Este enfoque es muy simple; sin embargo, ofrece perspectivas extremadamente poderosas. 
Claramente el concepto de la colusión entre proveedores o entre la demanda y la oferta es indistinguible en la mayoría de los casos porque, a partir de los datos, es muy difícil extraer las motivaciones para sostener este comportamiento ilícito.

Organizaremos este estudio en conjuntos de regresiones con indicadores de colusión. El primero contiene regresiones que consideran el porcentaje de ofertas falsas como un signo de colusión. El segundo considera los procesos adjudicados a un proveedor cuya primera y única oferta fue adjudicada en su primer intento. El tercero considera los procesos que fueron adjudicados sistemáticamente al mismo proveedor. El cuarto incluye regresiones que miden la colusión como la diferencia entre el precio estimado y el precio adjudicado.

\section{II.1. Discusión de indistinguibilidad en términos de análisis de datos}

\section{II.1.1. Principal análisis de componentes e interpretación ${ }^{8}$}

La creación de las variables proxy de colusión está sujeta a la indistinguibilidad del tipo de fenómeno. En algunos casos podría existir simultáneamente una colusión entre proveedores, y entre proveedor y comprador; por lo tanto, el proxy analizaría ambos fenómenos al mismo tiempo.

Sin embargo, dados los análisis gráficos representados en la figura 1, el primer componente podría ser interpretado como colusión entre proveedor y comprador (O-D). Por otro lado, el segundo componente es más cercano a las variables asociadas a la colusión entre proveedores. Esta representación nos da una idea de cómo agrupar las variables de la manifestación de colusión en dos grandes grupos (principales componentes) para obtener así mayores resultados. Por lo tanto, agregamos dos columnas finales que contienen esas regresiones de variables dependientes.

\section{II.2. Descripción de datos}

En esta sección describiremos las cifras agregadas analizadas en este documento. Básicamente, ilustramos con algunas tablas la información sobre el mercado de compras públicas que se relaciona con la colusión. Los grupos agregados son sector productivo, región, tamaño de empresa y posibilidad de la subcontratación.

8 Con el primer componente principal, explicamos el $31 \%$ de la variación. Al incluir los primeros y segundos componentes, explicamos el $56 \%$ de esta variación. 
Tabla 4

\begin{tabular}{lrccc}
\hline $\begin{array}{c}\text { Enterprise } \\
\text { size }\end{array}$ & $\begin{array}{c}\text { Dummy } \\
\text { bids }\end{array}$ & $\begin{array}{c}\text { Difference adj/ } \\
\text { eval price }\end{array}$ & $\begin{array}{c}\text { New Prov } \\
\text { awarded at 1st }\end{array}$ & $\begin{array}{c}\text { Excessive difference } \\
\text { adj/eval price (cases) }\end{array}$ \\
\hline BIG & $43,1 \%$ & $15,9 \%$ & $0,3 \%$ & $2,0 \%$ \\
MEDIUM & $175,3 \%$ & $21,7 \%$ & $5,3 \%$ & $14,0 \%$ \\
& $39,2 \%$ & $15,0 \%$ & $1,5 \%$ & $1,7 \%$ \\
MICRO & $140,9 \%$ & $20,9 \%$ & $12,1 \%$ & $13,1 \%$ \\
& $35,4 \%$ & $6,6 \%$ & $10,6 \%$ & $1,2 \%$ \\
SMALL & $119,3 \%$ & $15,7 \%$ & $30,8 \%$ & $10,8 \%$ \\
& $40,4 \%$ & $12,0 \%$ & $2,4 \%$ & $1,8 \%$ \\
Total & $131,0 \%$ & $20 \%$ & $15 \%$ & $13 \%$ \\
& $39,4 \%$ & $10,7 \%$ & $4,3 \%$ & $1,6 \%$ \\
\hline
\end{tabular}

(*) Mean first row, sd second row, all data is percentage of the total.

Tabla 5

\begin{tabular}{|c|c|c|c|c|}
\hline Region & $\begin{array}{l}\text { Dummy } \\
\text { bids }\end{array}$ & $\begin{array}{l}\text { Difference adj/ } \\
\text { eval price }\end{array}$ & $\begin{array}{c}\text { New Prov } \\
\text { awarded at 1st }\end{array}$ & $\begin{array}{l}\text { Excessive difference } \\
\text { adj/eval price (cases) }\end{array}$ \\
\hline \multirow[t]{2}{*}{ Antofagasta } & $30,8 \%$ & $9,8 \%$ & $5,2 \%$ & $0,9 \%$ \\
\hline & $119,4 \%$ & $18,9 \%$ & $22,2 \%$ & $9,5 \%$ \\
\hline \multirow[t]{2}{*}{ Araucanía } & $27,8 \%$ & $14,2 \%$ & $3,8 \%$ & $3,0 \%$ \\
\hline & $117,6 \%$ & $21,9 \%$ & $19,1 \%$ & $17,1 \%$ \\
\hline \multirow[t]{2}{*}{ Arica y Parinacota } & $27,7 \%$ & $10,6 \%$ & $4,0 \%$ & $2,4 \%$ \\
\hline & $83,0 \%$ & $18,7 \%$ & $19,6 \%$ & $15,4 \%$ \\
\hline \multirow[t]{2}{*}{ Atacama } & $38,5 \%$ & $11,8 \%$ & $4,2 \%$ & $2,1 \%$ \\
\hline & $128,4 \%$ & $20,3 \%$ & $20,1 \%$ & $14,3 \%$ \\
\hline \multirow[t]{2}{*}{ Aysén } & $26,4 \%$ & $11,0 \%$ & $5,5 \%$ & $1,7 \%$ \\
\hline & $83,2 \%$ & $20,5 \%$ & $22,8 \%$ & $13,1 \%$ \\
\hline \multirow[t]{2}{*}{ Bío-Bío } & $31,8 \%$ & $12,0 \%$ & $3,5 \%$ & $1,6 \%$ \\
\hline & $111,2 \%$ & $20,1 \%$ & $18,3 \%$ & $12,5 \%$ \\
\hline \multirow[t]{2}{*}{ Coquimbo } & $28,3 \%$ & $4,9 \%$ & $5,7 \%$ & $0,5 \%$ \\
\hline & $128,1 \%$ & $12,9 \%$ & $23,1 \%$ & $6,8 \%$ \\
\hline \multirow[t]{2}{*}{ Lib. Gral. Bdo. O’H } & $37,2 \%$ & $6,7 \%$ & $4,7 \%$ & $0,6 \%$ \\
\hline & $115,8 \%$ & $13,4 \%$ & $21,2 \%$ & $8,0 \%$ \\
\hline \multirow[t]{2}{*}{ Los Lagos } & $33,9 \%$ & $8,7 \%$ & $5,4 \%$ & $1,6 \%$ \\
\hline & $120,7 \%$ & $18,0 \%$ & $22,5 \%$ & $12,6 \%$ \\
\hline \multirow[t]{2}{*}{ Los Ríos } & $28,6 \%$ & $7,3 \%$ & $4,4 \%$ & $0,3 \%$ \\
\hline & $89,4 \%$ & $15,0 \%$ & $20,5 \%$ & $5,6 \%$ \\
\hline \multirow[t]{2}{*}{ Magallanes y Antártica } & $31,8 \%$ & $7,8 \%$ & $4,2 \%$ & $1,0 \%$ \\
\hline & $105,5 \%$ & $13,5 \%$ & $20,1 \%$ & $10,1 \%$ \\
\hline \multirow[t]{2}{*}{ Maule } & $27,4 \%$ & $8,2 \%$ & $4,2 \%$ & $1,2 \%$ \\
\hline & $94,3 \%$ & $16,8 \%$ & $20,1 \%$ & $10,9 \%$ \\
\hline \multirow[t]{2}{*}{ Metropolitana } & $65,1 \%$ & $11,4 \%$ & $2,6 \%$ & $1,7 \%$ \\
\hline & $245,6 \%$ & $19,4 \%$ & $15,8 \%$ & $13,0 \%$ \\
\hline \multirow[t]{2}{*}{ Tarapacá } & $33,4 \%$ & $15,4 \%$ & $3,9 \%$ & $3,3 \%$ \\
\hline & $100,3 \%$ & $22,4 \%$ & $19,3 \%$ & $17,9 \%$ \\
\hline \multirow[t]{2}{*}{ Valparaíso } & $34,5 \%$ & $9,2 \%$ & $3,8 \%$ & $1,2 \%$ \\
\hline & $140,9 \%$ & $17,2 \%$ & $19,0 \%$ & $10,7 \%$ \\
\hline \multirow[t]{2}{*}{ Total } & $43,5 \%$ & $10,7 \%$ & $3,7 \%$ & $1,6 \%$ \\
\hline & $173,8 \%$ & $19,0 \%$ & $18,8 \%$ & $12,6 \%$ \\
\hline
\end{tabular}

(*) Mean first row, sd second row, all data is percentage of the total. 
Tabla 6

\begin{tabular}{lcccc}
\hline $\begin{array}{c}\text { Possibility of } \\
\text { subcontracting }\end{array}$ & $\begin{array}{c}\text { Dummy } \\
\text { bids }\end{array}$ & $\begin{array}{c}\text { Difference } \\
\text { adj/eval price }\end{array}$ & $\begin{array}{c}\text { New Prov } \\
\text { awarded at 1st }\end{array}$ & $\begin{array}{c}\text { Excessive difference } \\
\text { adj/eval price (cases) }\end{array}$ \\
\hline No & $46 \%$ & $11 \%$ & $4 \%$ & $2 \%$ \\
& $183 \%$ & $19 \%$ & $19 \%$ & $13 \%$ \\
Yes & $30 \%$ & $10 \%$ & $4 \%$ & $1 \%$ \\
& $120 \%$ & $18 \%$ & $19 \%$ & $12 \%$ \\
Total & $44 \%$ & $11 \%$ & $4 \%$ & $2 \%$ \\
& $174 \%$ & $19 \%$ & $19 \%$ & $13 \%$ \\
\hline
\end{tabular}

(*) Mean first row, sd second row, all data is percentage of the total.

SOBRE LOS

DETERMINANTES

DE LA COLUSIÓN

EN LAS COMPRAS

PÚBLICAS: EL CASO

DE CHILE

THE DETERMINANTS

OF COLLUSION

IN PUBLIC

PROCUREMENT:

THE CASE OF CHILE

\begin{tabular}{|c|c|c|c|c|c|}
\hline Enterprise size & $\begin{array}{c}\text { Dummy } \\
\text { bids }\end{array}$ & $\begin{array}{c}\text { Difference adj/ } \\
\text { eval price }\end{array}$ & $\begin{array}{c}\text { New Prov } \\
\text { awarded at 1st }\end{array}$ & $\begin{array}{l}\text { Excessive difference } \\
\text { adj/eval price (cases) }\end{array}$ & $\begin{array}{l}\text { PROCUREMENT: } \\
\text { THE CASE OF CHILE }\end{array}$ \\
\hline \multirow[t]{2}{*}{ Food beverage } & $16 \%$ & $25 \%$ & $3 \%$ & $7 \%$ & \\
\hline & $74 \%$ & $27 \%$ & $17 \%$ & $26 \%$ & \\
\hline \multirow[t]{2}{*}{ Fuel lubricant } & $17 \%$ & $12 \%$ & $2 \%$ & $3 \%$ & \\
\hline & $69 \%$ & $21 \%$ & $13 \%$ & $16 \%$ & \\
\hline \multirow[t]{2}{*}{ Manufacturing comp \& supp } & $25 \%$ & $11 \%$ & $1 \%$ & $0 \%$ & \\
\hline & $62 \%$ & $15 \%$ & $10 \%$ & $0 \%$ & \\
\hline \multirow[t]{2}{*}{ Electronic comp \& supp } & $38 \%$ & $20 \%$ & $2 \%$ & $3 \%$ & \\
\hline & $90 \%$ & $19 \%$ & $12 \%$ & $16 \%$ & $\bar{c}$ \\
\hline \multirow[t]{2}{*}{ Construction Manuf comp \& supp } & $27 \%$ & $22 \%$ & $3 \%$ & $2 \%$ & \\
\hline & $77 \%$ & $24 \%$ & $17 \%$ & $16 \%$ & C \\
\hline \multirow[t]{2}{*}{ Education \& training services } & $25 \%$ & $1 \%$ & $12 \%$ & $0 \%$ & $\bar{z}$ \\
\hline & $100 \%$ & $6 \%$ & $33 \%$ & $0 \%$ & C \\
\hline \multirow{2}{*}{ Printing, Audio, Visual eq \& supp } & $32 \%$ & $13 \%$ & $1 \%$ & $1 \%$ & $\bar{C}$ \\
\hline & $93 \%$ & $22 \%$ & $9 \%$ & $11 \%$ & \\
\hline \multirow[t]{2}{*}{ Office acc \& supp } & $99 \%$ & $21 \%$ & $1 \%$ & $3 \%$ & \\
\hline & $349 \%$ & $24 \%$ & $7 \%$ & $16 \%$ & $\vec{\imath}$ \\
\hline \multirow[t]{2}{*}{ Medical eq, acc \& supp } & $71 \%$ & $23 \%$ & $1 \%$ & $2 \%$ & \\
\hline & $198 \%$ & $21 \%$ & $10 \%$ & $12 \%$ & $a$ \\
\hline \multirow[t]{2}{*}{ Laboratory eq } & $60 \%$ & $19 \%$ & $1 \%$ & $2 \%$ & \\
\hline & $180 \%$ & $22 \%$ & $9 \%$ & $14 \%$ & 9 \\
\hline \multirow[t]{2}{*}{ Public defense, safety eq \& supp } & $58 \%$ & $24 \%$ & $2 \%$ & $3 \%$ & \\
\hline & $143 \%$ & $23 \%$ & $13 \%$ & $16 \%$ & \\
\hline \multirow[t]{2}{*}{ Cleaning eq \& supp } & $76 \%$ & $16 \%$ & $1 \%$ & $1 \%$ & \\
\hline & $189 \%$ & $22 \%$ & $9 \%$ & $8 \%$ & $C$ \\
\hline \multirow[t]{2}{*}{ Sports eq, supp \& acc } & $53 \%$ & $18 \%$ & $2 \%$ & $2 \%$ & \\
\hline & $124 \%$ & $23 \%$ & $15 \%$ & $14 \%$ & \\
\hline \multirow[t]{2}{*}{ Tools \& gral mach } & $40 \%$ & $23 \%$ & $5 \%$ & $6 \%$ & \\
\hline & $122 \%$ & $24 \%$ & $21 \%$ & $23 \%$ & \\
\hline \multirow[t]{2}{*}{ Musical instr, games \& educ eq } & $35 \%$ & $12 \%$ & $2 \%$ & $1 \%$ & \\
\hline & $96 \%$ & $21 \%$ & $13 \%$ & $9 \%$ & r \\
\hline \multirow[t]{2}{*}{ Mining, drilling mach \& acc } & $19 \%$ & $9 \%$ & $6 \%$ & $1 \%$ & \\
\hline & $78 \%$ & $18 \%$ & $24 \%$ & $12 \%$ & 3 \\
\hline \multirow[t]{2}{*}{ Industrial process mach \& acc } & $30 \%$ & $22 \%$ & $4 \%$ & $2 \%$ & \\
\hline & $76 \%$ & $20 \%$ & $20 \%$ & $13 \%$ & \\
\hline \multirow[t]{2}{*}{ Farm, fish, forestry mach \& acc } & $19 \%$ & $8 \%$ & $6 \%$ & $0 \%$ & $\sum$ \\
\hline & $52 \%$ & $13 \%$ & $23 \%$ & $0 \%$ & \\
\hline \multirow[t]{2}{*}{ Construction, building mach \& acc } & $18 \%$ & $26 \%$ & $3 \%$ & $3 \%$ & \\
\hline & $53 \%$ & $25 \%$ & $18 \%$ & $16 \%$ & v \\
\hline \multirow[t]{2}{*}{ Power gen, distr, mach \& acc } & $52 \%$ & $22 \%$ & $3 \%$ & $3 \%$ & \\
\hline & $130 \%$ & $23 \%$ & $17 \%$ & $16 \%$ & \\
\hline \multirow[t]{2}{*}{ Mat handling, storage, mach acc \& supp } & $41 \%$ & $23 \%$ & $3 \%$ & $2 \%$ & \\
\hline & $106 \%$ & $22 \%$ & $17 \%$ & $13 \%$ & \\
\hline \multirow[t]{2}{*}{ Service Industr mach, eq \& supp } & $59 \%$ & $19 \%$ & $3 \%$ & $4 \%$ & \\
\hline & $124 \%$ & $26 \%$ & $16 \%$ & $19 \%$ & $z$ \\
\hline \multirow[t]{2}{*}{ Live plant/animal mat, acc \& supp } & $34 \%$ & $12 \%$ & $8 \%$ & $1 \%$ & \\
\hline & $75 \%$ & $18 \%$ & $27 \%$ & $10 \%$ & \\
\hline
\end{tabular}




\begin{tabular}{|c|c|c|c|c|}
\hline \multirow[t]{2}{*}{ Paper mat \& products } & $56 \%$ & $22 \%$ & $1 \%$ & $4 \%$ \\
\hline & $121 \%$ & $27 \%$ & $10 \%$ & $20 \%$ \\
\hline \multirow[t]{2}{*}{ Mineral \& textile, plant $\&$ animal mat } & $26 \%$ & $14 \%$ & $4 \%$ & $1 \%$ \\
\hline & $83 \%$ & $15 \%$ & $19 \%$ & $7 \%$ \\
\hline \multirow[t]{2}{*}{ Pharmaceutical products } & $104 \%$ & $20 \%$ & $0 \%$ & $2 \%$ \\
\hline & $374 \%$ & $21 \%$ & $4 \%$ & $15 \%$ \\
\hline \multirow[t]{2}{*}{ Furniture and Furnishings } & $55 \%$ & $17 \%$ & $2 \%$ & $1 \%$ \\
\hline & $155 \%$ & $20 \%$ & $14 \%$ & $12 \%$ \\
\hline \multirow[t]{2}{*}{ Domestic appliances \& acc } & $72 \%$ & $21 \%$ & $1 \%$ & $2 \%$ \\
\hline & $152 \%$ & $23 \%$ & $10 \%$ & $14 \%$ \\
\hline \multirow[t]{2}{*}{ Organizations \& clubs } & $116 \%$ & $1 \%$ & $12 \%$ & $0 \%$ \\
\hline & $11 \%$ & $5 \%$ & $32 \%$ & $0 \%$ \\
\hline \multirow[t]{2}{*}{ Politics and Civic Affairs serv } & $13 \%$ & $1 \%$ & $8 \%$ & $0 \%$ \\
\hline & $87 \%$ & $7 \%$ & $28 \%$ & $5 \%$ \\
\hline \multirow[t]{2}{*}{ Published Products } & $77 \%$ & $18 \%$ & $2 \%$ & $3 \%$ \\
\hline & $199 \%$ & $26 \%$ & $13 \%$ & $17 \%$ \\
\hline \multirow[t]{2}{*}{ Jewlery prod } & $7 \%$ & . & $7 \%$ & $0 \%$ \\
\hline & $27 \%$ & . & $27 \%$ & $0 \%$ \\
\hline \multirow[t]{2}{*}{ Chemical prod } & $24 \%$ & $14 \%$ & $1 \%$ & $1 \%$ \\
\hline & $72 \%$ & $23 \%$ & $8 \%$ & $12 \%$ \\
\hline \multirow[t]{2}{*}{ Rubber mat } & $20 \%$ & $22 \%$ & $5 \%$ & $5 \%$ \\
\hline & $45 \%$ & $21 \%$ & $23 \%$ & $23 \%$ \\
\hline \multirow[t]{2}{*}{ Baggage $\%$ pers care prod } & $46 \%$ & $19 \%$ & $1 \%$ & $2 \%$ \\
\hline & $133 \%$ & $23 \%$ & $11 \%$ & $13 \%$ \\
\hline \multirow[t]{2}{*}{ Healthcare Services } & $18 \%$ & $3 \%$ & $9 \%$ & $0 \%$ \\
\hline & $114 \%$ & $10 \%$ & $28 \%$ & $6 \%$ \\
\hline \multirow[t]{2}{*}{ Farm, fish, forestry contract serv } & $26 \%$ & $2 \%$ & $7 \%$ & $0 \%$ \\
\hline & $193 \%$ & $8 \%$ & $25 \%$ & $4 \%$ \\
\hline Engineering \& research serv & $76 \%$ & $9 \%$ & $3 \%$ & $1 \%$ \\
\hline & $156 \%$ & $20 \%$ & $17 \%$ & $10 \%$ \\
\hline Public utilities \& public sector serv & $31 \%$ & $10 \%$ & $1 \%$ & $1 \%$ \\
\hline & $91 \%$ & $16 \%$ & $10 \%$ & $11 \%$ \\
\hline Construction maintenance serv & $7 \%$ & $0 \%$ & $10 \%$ & $0 \%$ \\
\hline & $45 \%$ & $4 \%$ & $30 \%$ & $0 \%$ \\
\hline Personal and Domestic serv & $20 \%$ & $9 \%$ & $5 \%$ & $1 \%$ \\
\hline & $86 \%$ & $17 \%$ & $23 \%$ & $10 \%$ \\
\hline Public defense, safety services & $16 \%$ & $6 \%$ & $4 \%$ & $0 \%$ \\
\hline & $52 \%$ & $13 \%$ & $20 \%$ & $0 \%$ \\
\hline Industrial Cleaning serv & $24 \%$ & $22 \%$ & $4 \%$ & $2 \%$ \\
\hline & $78 \%$ & $23 \%$ & $20 \%$ & $15 \%$ \\
\hline Mining and oil and gas serv & $30 \%$ & $12 \%$ & $6 \%$ & $0 \%$ \\
\hline & $91 \%$ & $14 \%$ & $23 \%$ & $0 \%$ \\
\hline Industrial prod manuf serv & $37 \%$ & $10 \%$ & $7 \%$ & $1 \%$ \\
\hline & $93 \%$ & $17 \%$ & $25 \%$ & $12 \%$ \\
\hline Transport, storage \& mail serv & $14 \%$ & $0 \%$ & $13 \%$ & $0 \%$ \\
\hline & $40 \%$ & $0 \%$ & $33 \%$ & $0 \%$ \\
\hline Travel, food, lodging \& entert serv & $20 \%$ & $14 \%$ & $5 \%$ & $1 \%$ \\
\hline & $73 \%$ & $17 \%$ & $22 \%$ & $9 \%$ \\
\hline Editorial, graphic \& fine art design & $25 \%$ & $9 \%$ & $6 \%$ & $2 \%$ \\
\hline & $81 \%$ & $18 \%$ & $23 \%$ & $15 \%$ \\
\hline Financial \& insurance serv & $30 \%$ & $8 \%$ & $6 \%$ & $2 \%$ \\
\hline & $96 \%$ & $18 \%$ & $24 \%$ & $13 \%$ \\
\hline Environmental serv & $20 \%$ & $15 \%$ & $10 \%$ & $1 \%$ \\
\hline & $69 \%$ & $26 \%$ & $30 \%$ & $11 \%$ \\
\hline Business, manag, proff $\&$ admin serv & $23 \%$ & $3 \%$ & $10 \%$ & $1 \%$ \\
\hline & $95 \%$ & $10 \%$ & $30 \%$ & $7 \%$ \\
\hline Distrib, condit systems, eq \& comp & $37 \%$ & $20 \%$ & $3 \%$ & $3 \%$ \\
\hline & $121 \%$ & $22 \%$ & $16 \%$ & $18 \%$ \\
\hline Electric systems, light acc $\&$ supp & $39 \%$ & $24 \%$ & $2 \%$ & $4 \%$ \\
\hline & $92 \%$ & $27 \%$ & $12 \%$ & $19 \%$ \\
\hline IT broadcast $\&$ telecomunication & $47 \%$ & $18 \%$ & $1 \%$ & $1 \%$ \\
\hline & $142 \%$ & $20 \%$ & $11 \%$ & $11 \%$ \\
\hline Vehicles, acc, comp & $29 \%$ & $10 \%$ & $2 \%$ & $1 \%$ \\
\hline & $114 \%$ & $18 \%$ & $14 \%$ & $8 \%$ \\
\hline Total & $\begin{array}{r}44 \% \\
2 \%\end{array}$ & $\begin{array}{l}11 \% \\
19 \%\end{array}$ & $\begin{array}{r}4 \% \\
19 \%\end{array}$ & $\begin{array}{r}2 \% \\
13 \%\end{array}$ \\
\hline
\end{tabular}

(*) Mean first row, sd second row, all data is percentage of the total. 


\section{II.3. Regresiones de colusión}

\section{II.3.1. El modelo}

Ejecutamos siete conjuntos de regresiones de variables dependientes nucleares $(\mathrm{k}=1,2, \ldots, 7)$ :

$$
\text { Coll k/i }=\infty \mathrm{k}+\beta \mathrm{j} / \mathrm{kXj} / \mathrm{i}+\varepsilon \mathrm{k} / \mathrm{i}
$$

Donde la variable dependiente representa la colusión a través de:

- Coll 1/i Adjudicada al primer oferente $(0,1)$

- Coll 2/i Ganadores repetidos (cantidad de licitaciones adjudicadas por la misma unidad de compras)

- Coll 3/i Ofertas falsas (cantidad de ofertas mayores o iguales 2 veces el precio adjudicado)

- Coll 4/i Distorsiones de precio, precios adjudicados excesivamente altos (como porcentaje del precio estimado)

- Coll 5/i Distorsiones de precio, precios adjudicados excesivamente altos (cuando Coll 4/i es mayor o igual a 50\%.)

- Coll 6/i Primer componente principal ${ }^{9}(\mathrm{O}-\mathrm{D})$

- Coll 7/i Segundo componente principal (O-O)

Las variables independientes se describen a continuación $(j=1, \ldots 80)$ :

- X1 Subcontratación $(0,1)$

- $\mathrm{X}_{2}$ Fecha de publicación (registros)

- $X_{3}$ Cantidad de ofertas

- X4 Cantidad de proveedores adjudicados

- X5 Adjudicaciones múltiples $(0,1)$

- X6 Monto total de la licitación

- X7 Pequeña empresa $(0,1)$

- X8 Mediana empresa

- X9Gran empresa

- X10 Días de decisión

- X11 Exceso de ofertas (1 si el número de oferta $>=9,0$ )

- X12 Cantidad de unidades de compras del organismo comprador (centralización)

- X13-26 Variables ficticias geográficas regionales

9 Solamente para el caso en que incluimos la clasificación de productos y servicios. El primer componente principal estaría relacionado con la colusión entre un proveedor y un comprador (O-D), mientras que el segundo sería colusión entre proveedores (O-O). Sin embargo, esta distinción no está totalmente clara. La interpretación será financiada posteriormente.

SOBRE LOS

DETERMINANTES

DE LA COLUSIÓN

EN LAS COMPRAS

PÚBLICAS: EL CASO

DE CHILE

THE DETERMINANTS

\section{OF COLLUSION}

IN PUBLIC

PROCUREMENT:

THE CASE OF CHILE 
- X27 Tamaño de la licitación (1 si está dentro del rango 100. 1000 UTM $^{10}$ )

- X28 Tamaño de la licitación (1 si > 1000 UTM)

- X30-84 Clasificación de producto o servicio (55 UN nivel 1)

Ejecutamos una serie de regresiones de forma reducida ${ }^{11}$; dichas regresiones son dependientes de las características de la variable dependiente. Si la variable no es binaria, simplemente ejecutamos regresiones $\mathrm{MCO}$ al recopilar todos los datos disponibles. Por el contrario, si la variable es binaria, ejecutamos Probit y Logit para comparar cómo ambos modelos calzan con los datos.

Este tipo de metodología está en línea con la literatura más reciente ${ }^{12}$. La mayoría de los modelos son bastante nuevos y buscan detectar patrones sutiles en los datos. Nuestro caso es similar; sin embargo, nuestra definición de colusión es un poco diferente. Definimos "variables dependientes" como posibilidades de colusión, al reconocer el hecho de que la gran mayoría del tiempo la colusión no puede observarse o tiene un importante componente invisible. Otra diferencia es el sujeto de nuestro análisis: en vez de trabajar con las ofertas como datos atómicos individuales, utilizamos el proceso de licitación y sus características. Por eso utilizamos el estándar MCO, Probit y Logit en datos de corte transversal, pero de todas maneras controlamos por tendencias de tiempo con la variable independiente fecha de publicación. La siguiente tabla resume el primer conjunto de regresiones.

La primera y segunda columna de este conjunto de regresiones incluye un modelo en que el patrón de colusión está relacionado con el hecho de que hay nuevos proveedores que han sido adjudicados en la primera oportunidad en que ingresan una oferta. La variable dependiente es binaria, y ejecutamos un modelo Probit y Logit para este conjunto de regresiones. La tercera columna ofrece una estimación de la colusión cuando hay ganadores repetidos: la variable toma el valor de la cantidad de procesos adjudicados al mismo proveedor por una unidad de compra específica. La cuarta columna representa las ofertas falsas. En este caso, la variable considera el valor de la cantidad de ofertas que son más del doble del precio adjudicado — solo para el caso de procesos adjudicados a un solo proveedor- - La quinta columna representa la regresión cuando la

10 Se trata de la Unidad Tributaria Mensual, con un valor aproximado de US\$\$57 a diciembre de 2008. 11 Podríamos haber estimado un modelo estructural. Esto resolvería preguntas relacionadas con el carácter óptimo de los precios de reserva o los precios inflados impuestos por los oferentes. Dejamos este tipo de estimación para investigaciones futuras por dos razones principales: primero, nuestro análisis se centra exclusivamente en el aspecto de la colusión; segundo, la naturaleza de corte transversal de nuestros datos y la complejidad del entorno de la generación de datos sugieren evitar un enfoque estructural complejo.

12 Como en Bajari, Patrick y Garret Summers. "Detecting collusion in procurement auctions", 2002, entre otros. 


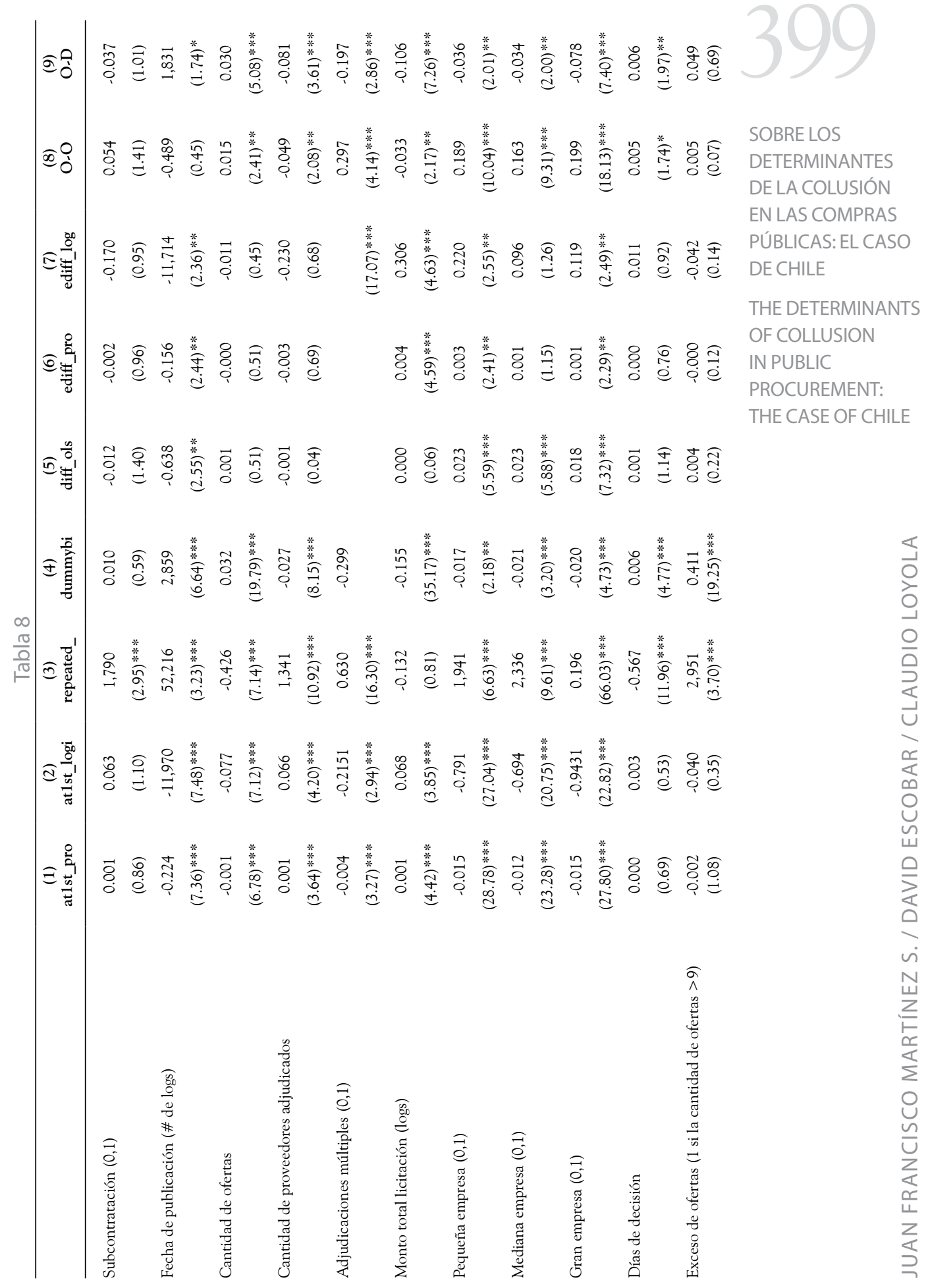




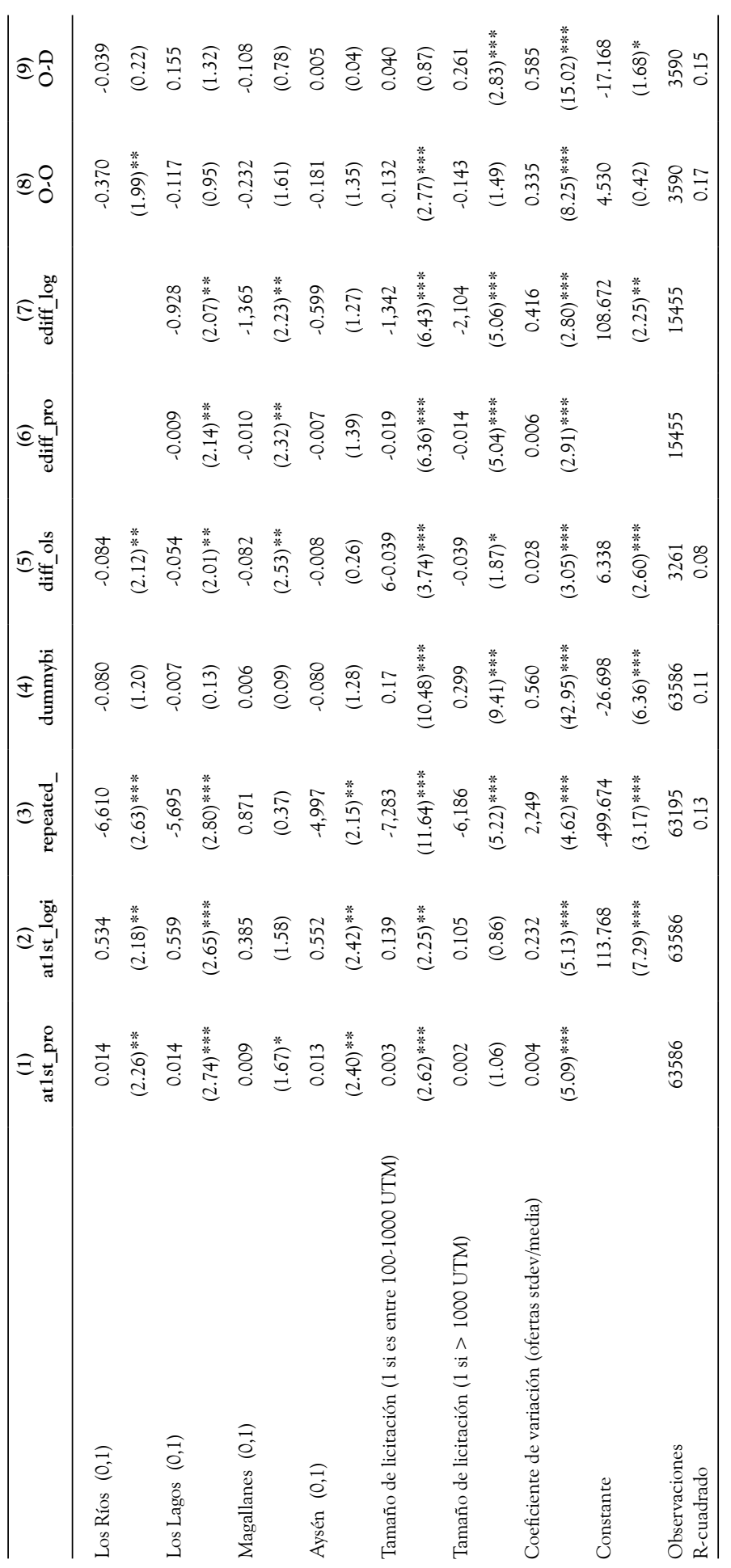

101

SOBRE LOS

DETERMINANTES

DE LA COLUSIÓN

EN LAS COMPRAS

PÚBLICAS: EL CASO

DE CHILE

THE DETERMINANTS

OF COLLUSION

IN PUBLIC

PROCUREMENT:

THE CASE OF CHILE 
variable dependiente es la diferencia entre el precio adjudicado y el precio estimado, como un porcentaje del precio estimado del bien o servicio. La sexta columna mide la colusión como la excesiva diferencia entre el precio estimado y el precio adjudicado; la variable establece un valor de "1" si la diferencia del porcentaje de la quinta columna es mayor a un 50\% de lo estimado.

Muchos de los resultados están bastantes relacionados a la intuición económica; no obstante, algunos requieren una segunda consideración, principalmente por el efecto de las múltiples variables simultáneas.

\section{II.3.2. Resultados de columnas}

Los coeficientes de la primera y segunda columna (a $1^{\text {a }}$ Probit y Logit) implican que este tipo de fenómeno de colusión es decreciente en el tiempo. Asimismo, implican que las empresas más pequeñas tienen más probabilidades de estar asociadas a la colusión entre proveedor y comprador (O-D). Claramente - y consistentemente con la literatura-, mientras más ofertas reciba una licitación, tiene menos probabilidades de presentar colusión; sin embargo, mientras más proveedores son adjudicados, mayores son las posibilidades de colusión. Esto se debe a un hecho conocido: es más fácil que existan acuerdos ocultos cuando el universo de proveedores asociados a una licitación es mayor, ya que existen menos probabilidades de detectar el ilícito. El mismo argumento funciona para el esquema de adjudicaciones múltiples: si presenta adjudicaciones múltiples, entonces las posibilidades de colusión son menores. Además, las regiones más predispuestas a este fenómeno son aquellas que están ubicadas en áreas geográficas remotas.

La tercera columna examina la existencia de colusión donde el proveedor es adjudicado repetidamente por la misma unidad de compras. Esto puede estar relacionado con ambos tipos de colusión: entre proveedores, o entre proveedor y comprador. Sin embargo, dado nuestro análisis anterior de los componentes principales, esto probablemente tiene que ver más con la colusión $(\mathrm{O}-\mathrm{O})$. No es sorpresa que esta variable esté vinculada a la opción de subcontratar el bien o servicio, y al esquema de adjudicaciones múltiples o combinatorio. El efecto es altamente significativo, tanto estadística como económicamente, para ambas variables. Esto se explica principalmente porque, mientras más proveedores son adjudicados en una licitación, es más fácil ocultar cualquier irregularidad $^{13}$, particularmente al ser adjudicado en forma repetida. Este tipo de colusión afecta sobre todo a las grandes empresas. En este caso, las variables inversamente relacionadas a la colusión son la cantidad de ofertas — que no es lo mismo que la cantidad de oferentes_ y la cantidad de 
unidades de compras en el organismo comprador. Estos hechos se explican porque, a mayor cantidad de ofertas, mejor será la competencia, y, a mayor cantidad de unidades de compras, mejor será la centralización de la unidad de compras, que debe procurar un control más estricto.

La cuarta columna está vinculada a la colusión entre proveedores (O-O). De alguna manera, los resultados van en contra de la intuición, pero una segunda consideración clarifica esta intuición. En este caso, los resultados son contrarios a los de las primeras tres columnas; el valor de las variables cantidad de ofertas y adjudicaciones es lo opuesto, pero sigue siendo significativo. Esto se explica porque, a mayor cantidad de ofertas, mayores son las probabilidades de tener ofertas falsas, ya que el alcance es mayor. Por otro lado, el hecho de que menos proveedores sean adjudicados facilita la detección de estas ofertas. Por esa razón, los vendedores se anticipan a la acción del comprador y evitan así este comportamiento.

Las columnas quinta, sexta y séptima, respectivamente, explican las diferencias entre precios adjudicados y estimados por un porcentaje o una variable ficticia. Queda claro que, mientras sea mayor la diferencia, existen mayores posibilidades de colusión. Los resultados son similares a los que se presentan en el párrafo anterior. Sin embargo, la principal diferencia es la cantidad de unidades de compras, que no tiene un efecto claro sobre las posibilidades de colusión. Esto se explica porque el fenómeno del precio excesivo de adjudicación está ocurriendo en todo el territorio, y ello afecta aun más a las entidades con mayor cantidad de compras. Además, a diferencia de la cuarta columna, utilizando la variable de la fecha podemos inferir que el efecto de este tipo de colusión está disminuyendo con el tiempo.

Un hecho notable es que la cantidad de unidades de compras de un organismo es tratada como un proxy de independencia del gobierno central u organismo público con resultados significativos. Mientras más unidades de compras posea, menos independiente será el organismo. En este sentido, es bastante impresionante que esta variable sea estadísticamente significativa y negativa respecto a la posibilidad de colusión en la mayoría de los casos. Esto podría ser porque, mientras más independiente sea el organismo, menos puede ser supervisado para asegurar su transparencia. En este caso, se debe realizar un tratamiento especial con licitaciones publicadas por municipalidades o pequeñas provincias. Por otro lado, este resultado es mínimo, o hasta positivo, si se consideran las diferencias en las regresiones de precios (columnas 5, 6 y 7). Esto se podría explicar porque las instituciones centralizadas y más grandes, con muchas unidades de compras y gastos, están más expuestas a sufrir colusión $(\mathrm{O}-\mathrm{O})$ en términos de diferencias en los precios, claramente debido al hecho de que, mientras más grande sea un organismo, tendrá 
más procesos de licitación que pueden ser oportunidades de obtener dinero extra para todo tipo de empresas, especialmente para las grandes.

Otro hecho notable es que la presencia de un subconjunto de problemas de colusión (columnas 1, 2 y 4) es más probable en las pequeñas empresas, pero al mismo tiempo en licitaciones medianas o grandes. En cambio, las licitaciones de menor tamaño, en las cuales presentan ofertas las grandes empresas, son más susceptibles de sufrir de otros tipos de colusión, según las columnas 3, 5, 6 y 7. Es decir, cuando el precio anormal o repetición del ganador son fuentes de ganancias ilícitas, las medianas y grandes empresas anticipan que los órganos supervisores tendrán mayores dificultades cuando los procesos involucran grandes montos.

Finalmente, el efecto geográfico es poco ambiguo: las licitaciones son más susceptibles a la colusión (O-D) cuando la región está más distante del centro del país. Por el contrario, la colusión $(\mathrm{O}-\mathrm{O})$ es más factible en el centro el país - cerca de los polos de desarrollo- . Este efecto es mixto cuando la colusión (O-O) genera diferencias en los precios.

\section{I.4. Análisis y especificaciones alternativos}

En el conjunto anterior de regresiones, generalmente se mantuvieron los resultados previos. Mejoramos una parte de la variabilidad al sumar más variables explicativas. Pero la mayor mejora radica en que aquí se puede observar cuáles de los rubros o sectores de negocios están más afectados por qué tipos de colusión.

Vemos, al igual que en las opiniones y hallazgos de los practicantes, que los principales rubros que presentan colusión son materiales y servicios de construcción, servicios de consultoría educacional y administrativa, y equipos farmacéuticos, médicos y de laboratorio, entre otros. En los siguientes párrafos describiremos en mayor detalle estos resultados.

En primer lugar, para licitaciones en que un proveedor nuevo es adjudicado al presentar su primera oferta (columnas 1 y 2), los rubros afectados por la colusión son servicios educacionales y de capacitación; maquinaria y accesorios para la minería; accesorios y maquinaria derivados de animales o plantas — una amplia variedad de productos para y a partir de animales y plantas son utilizados para fines medioambientales y de conservación-; productos para pequeñas organizaciones comunitarias, consultorías políticas y sociales profesionales; servicios públicos de comida y salud; servicios de limpieza industrial; servicios de perforación minera; servicios de viajes; y servicios de consultoría medioambiental.

En segundo lugar, para el tipo de colusión en que el ganador es extremadamente afortunado (columna 3), los rubros más afectados son insumos de construcción y abastecimiento; equipos y accesorios médicos; 

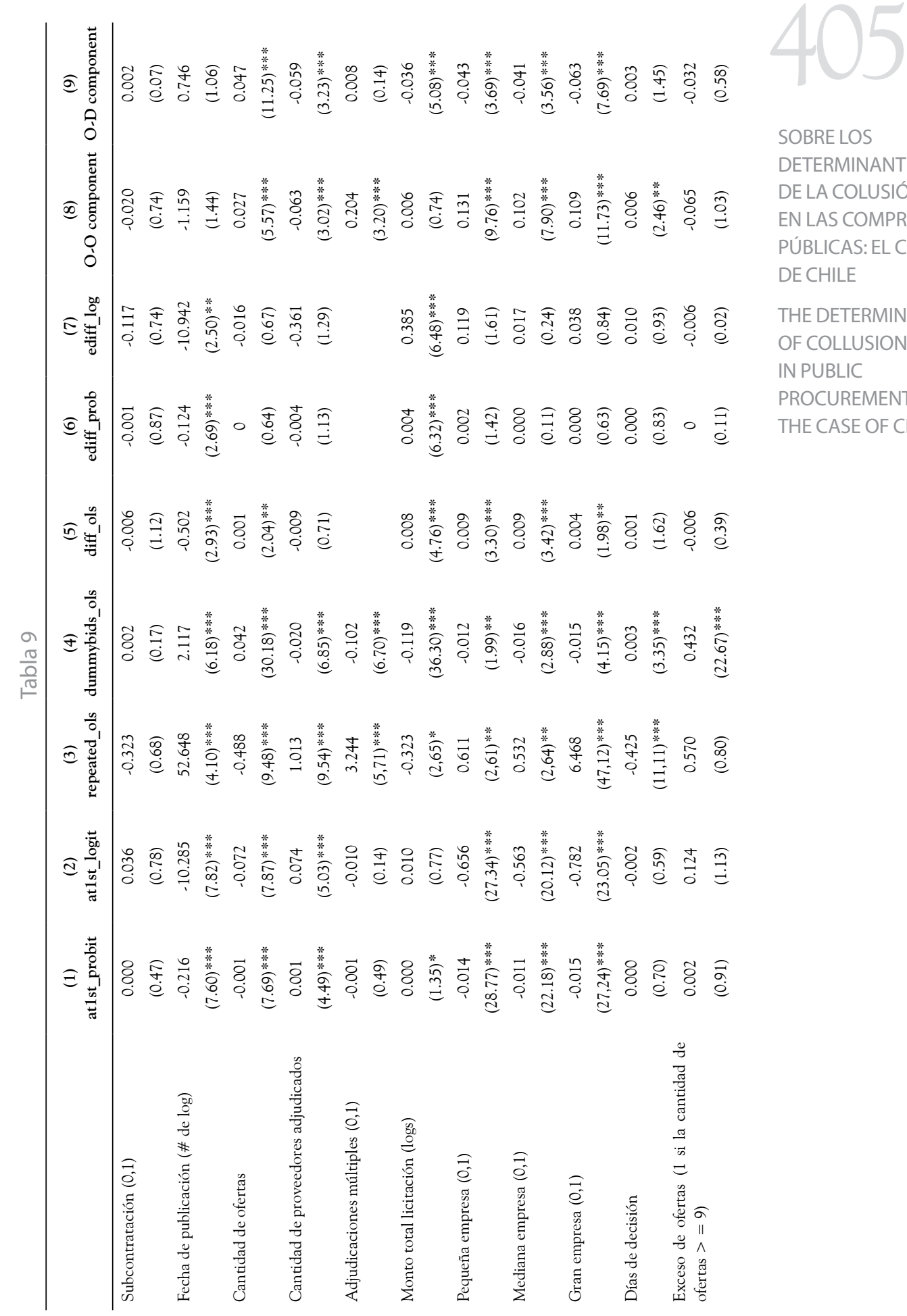

SOBRE LOS

DETERMINANTES

DE LA COLUSIÓN

EN LAS COMPRAS

PÚBLICAS: EL CASO

DE CHILE

THE DETERMINANTS

OF COLLUSION

IN PUBLIC

PROCUREMENT:

THE CASE OF CHILE 


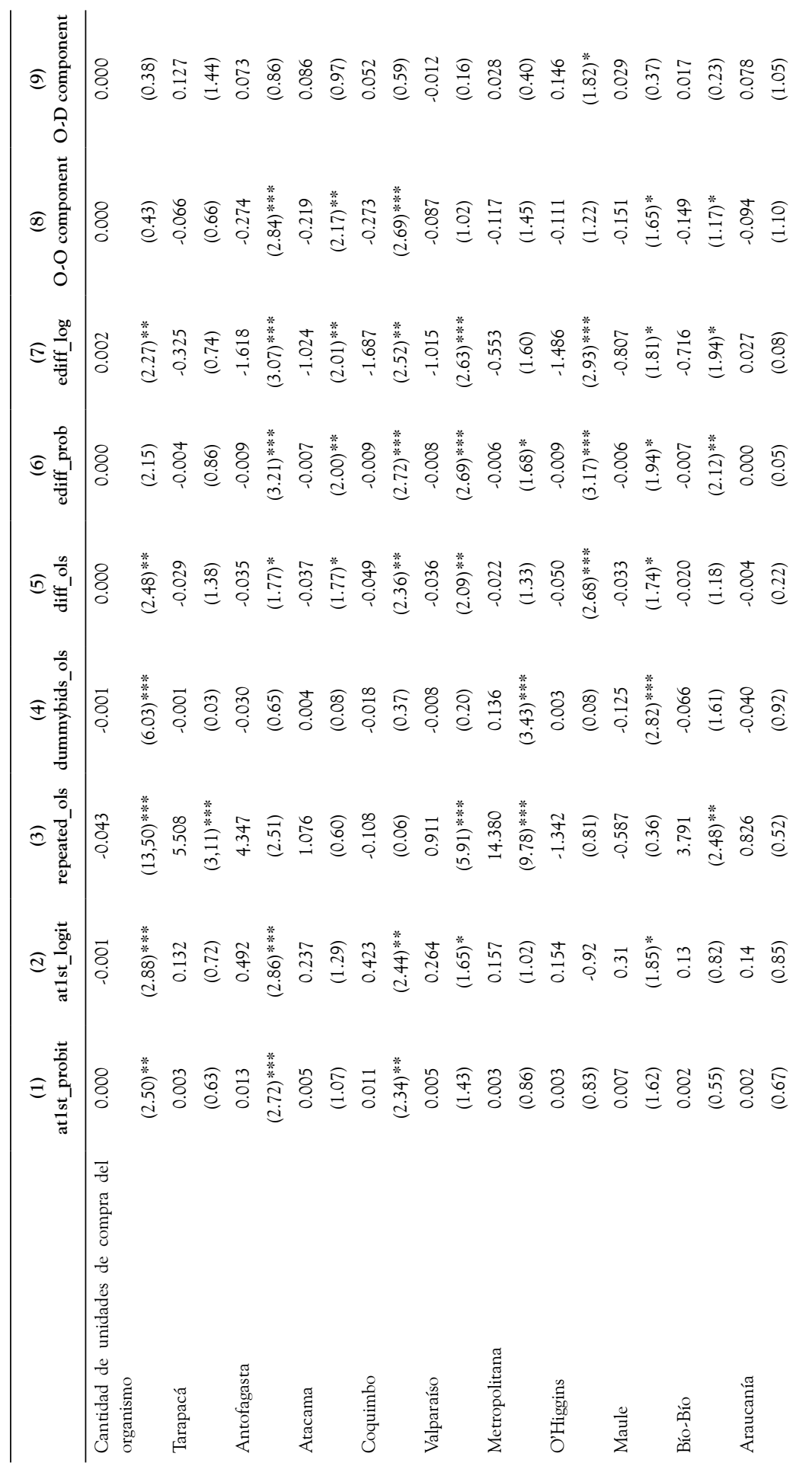




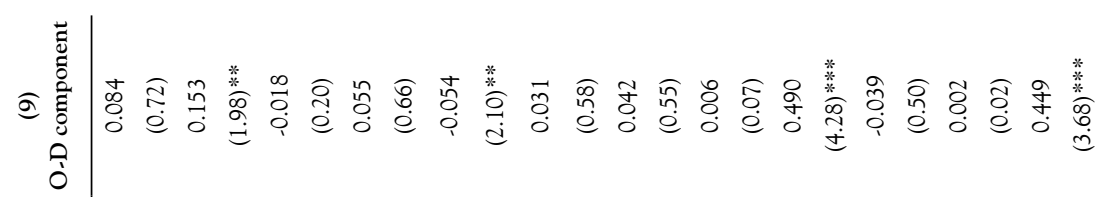

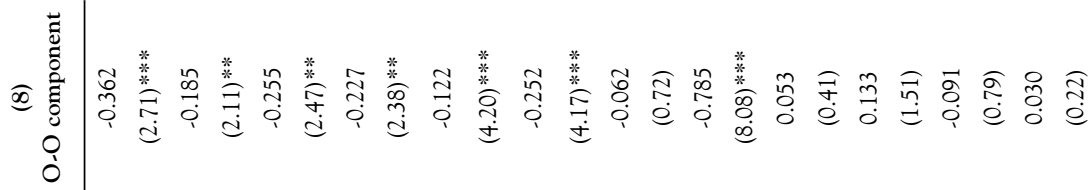

SOBRE LOS

DETERMINANTES

DE LA COLUSIÓN

EN LAS COMPRAS

PÚBLICAS: EL CASO

DE CHILE

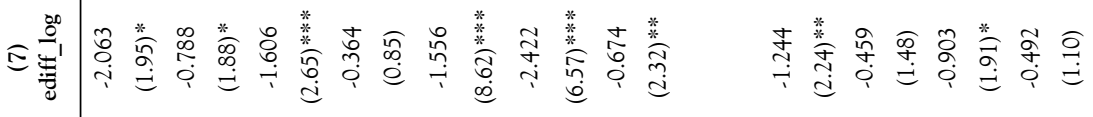

THE DETERMINANTS

of COLLUSION

IN PUBLIC

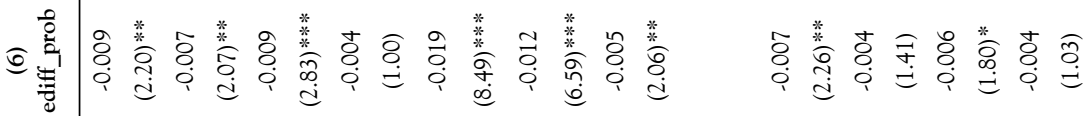

PROCUREMENT:

THE CASE OF CHILE

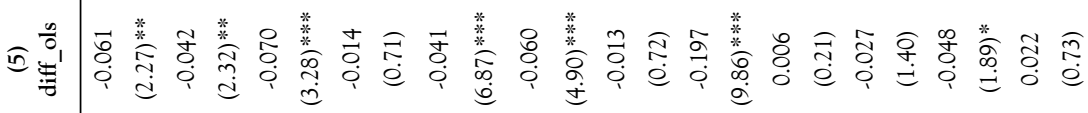

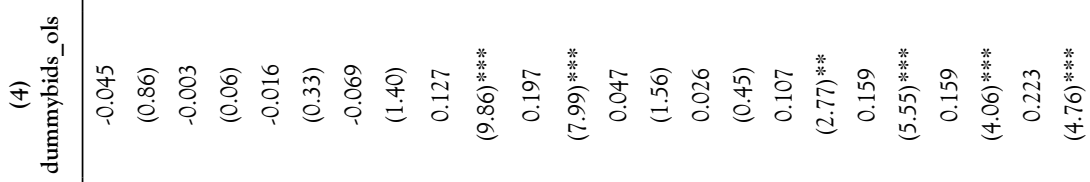

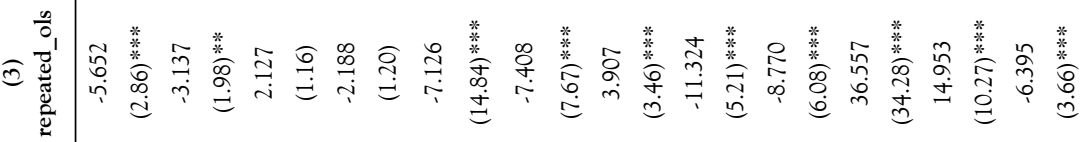

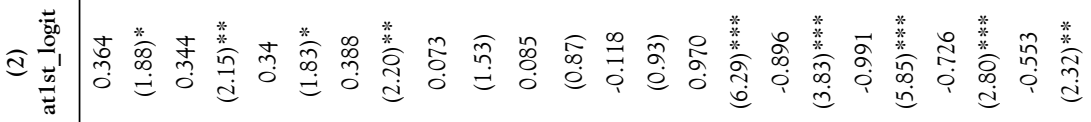

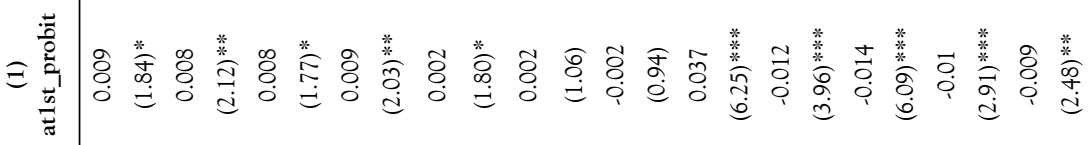



Derecho PUCP, N 66, 2011 / ISSN 0251-3420 


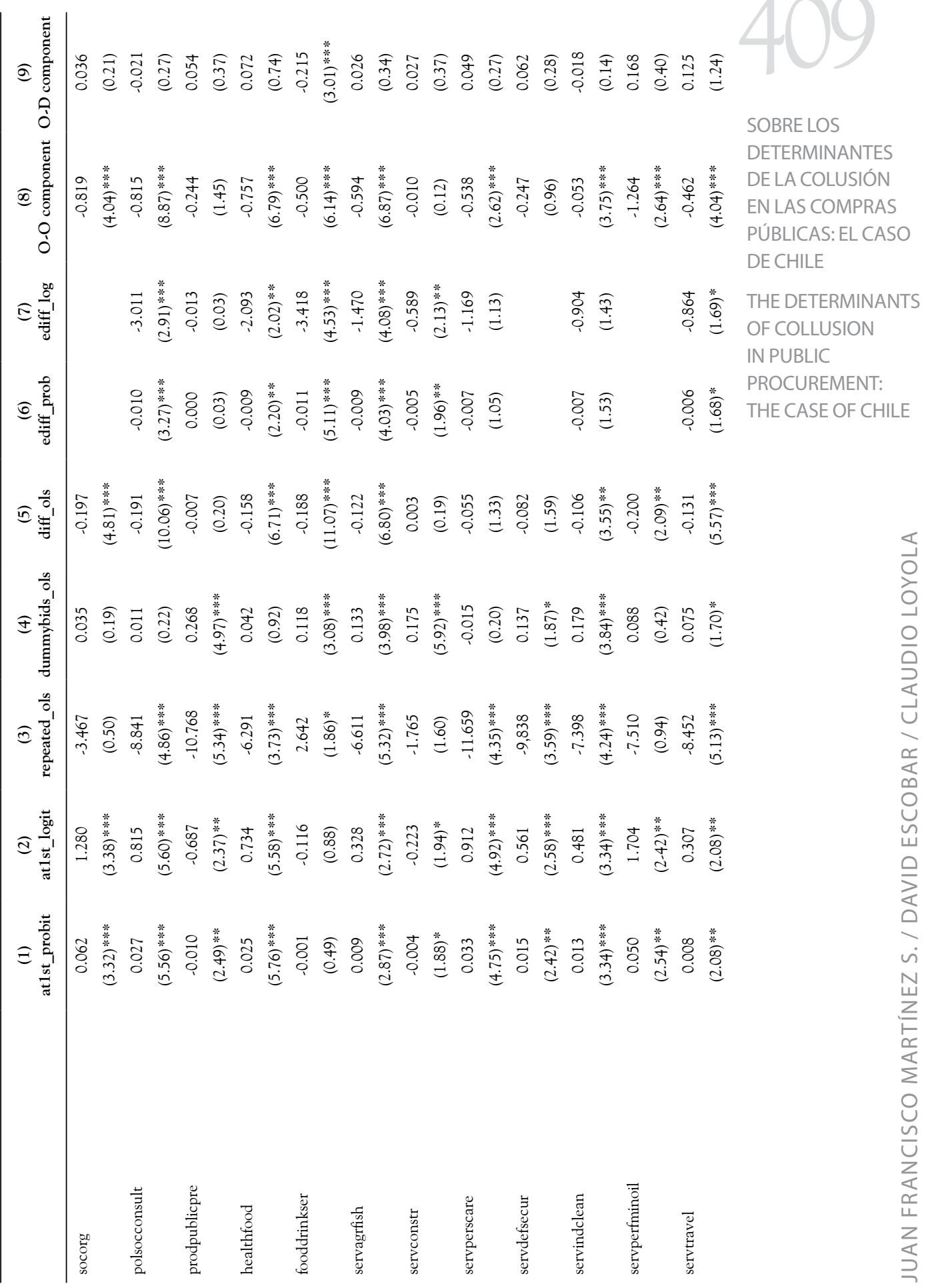




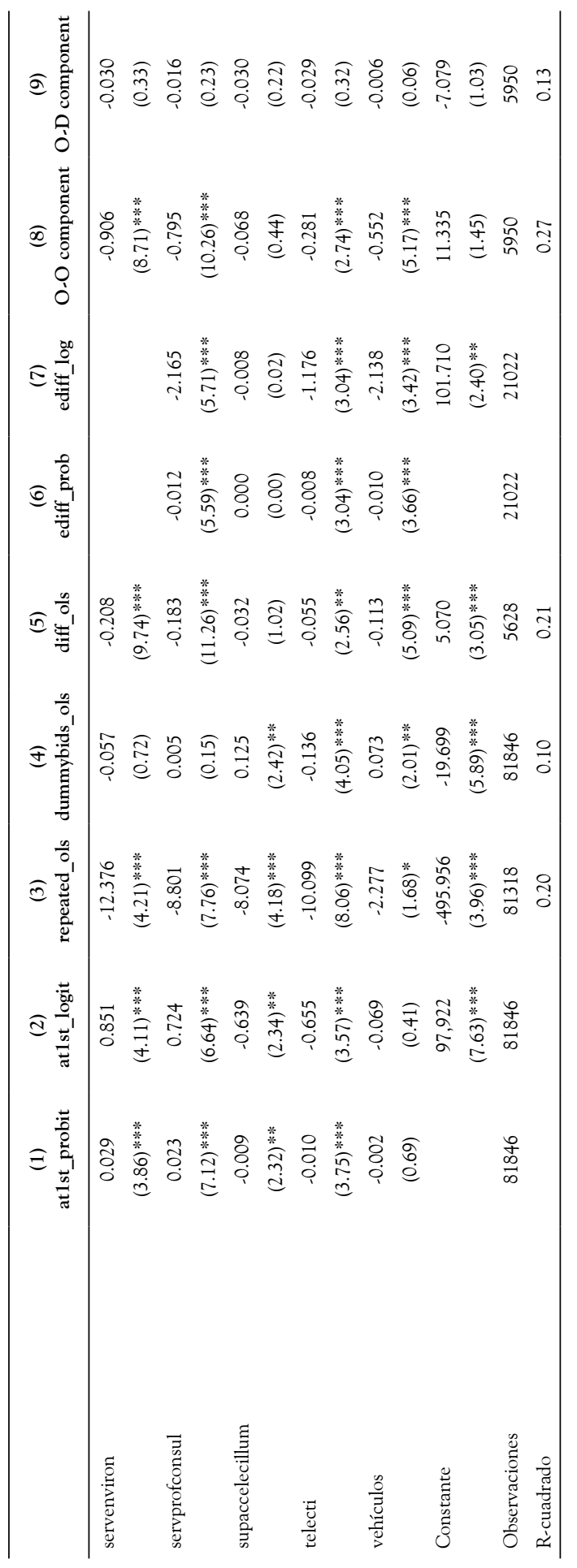

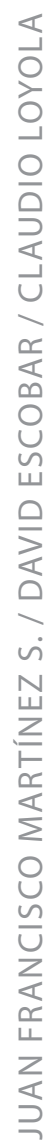




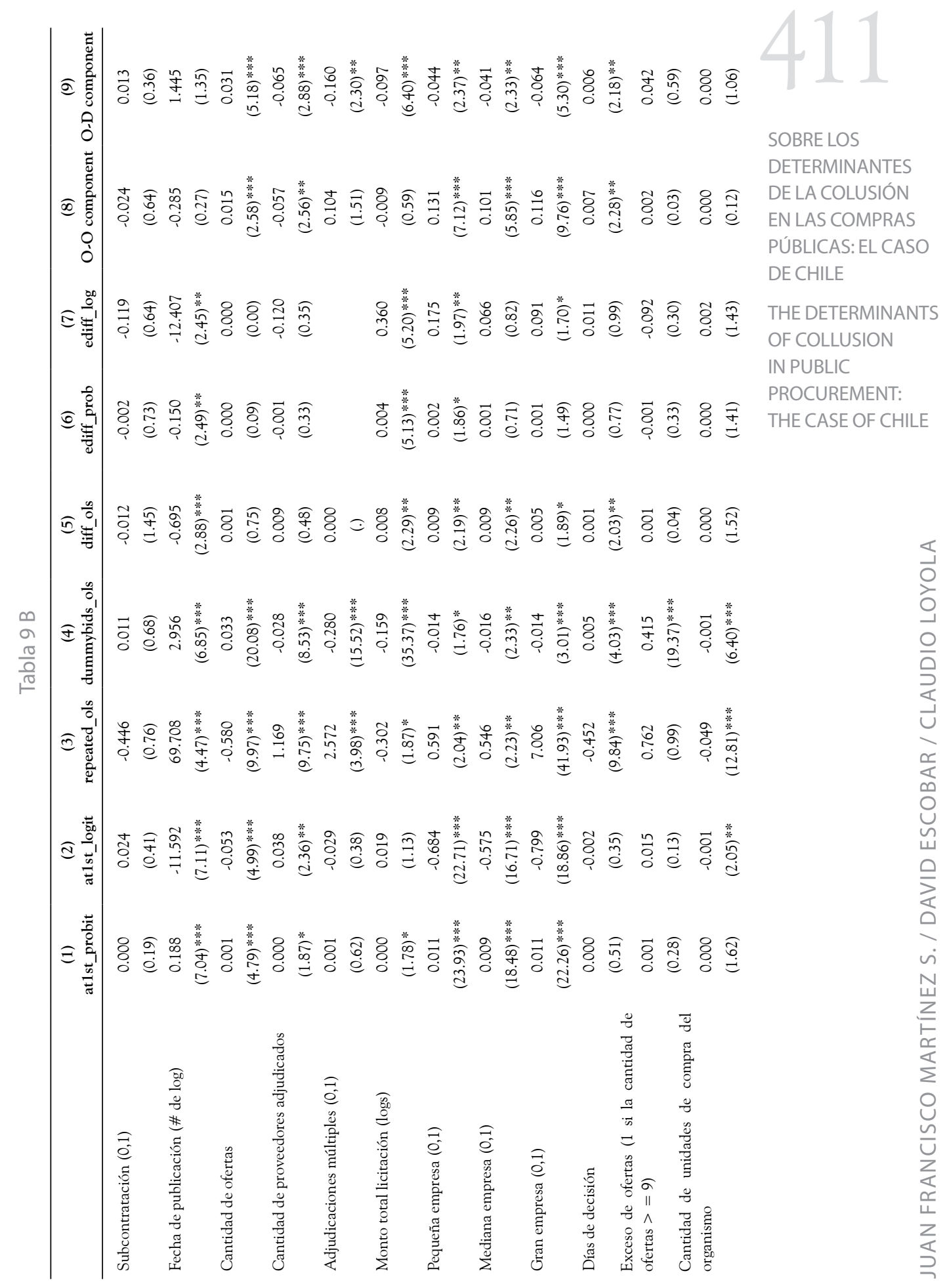




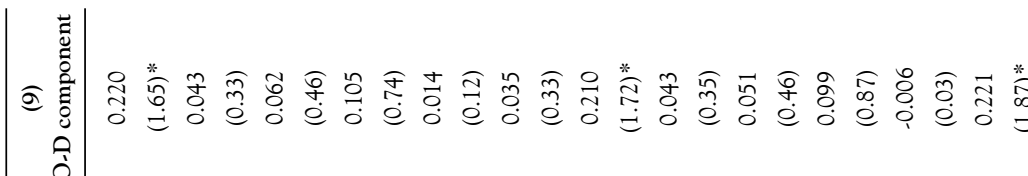

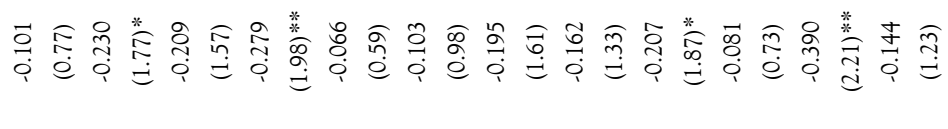

害

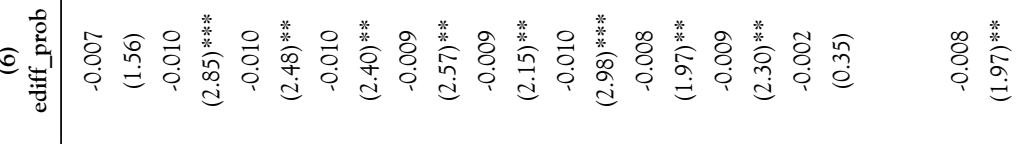

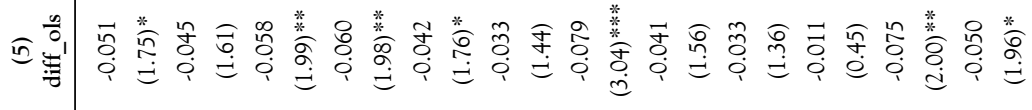

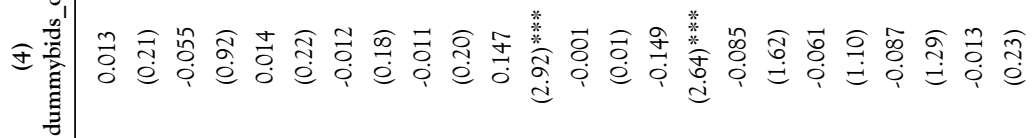

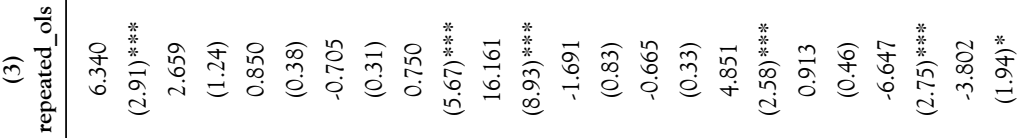

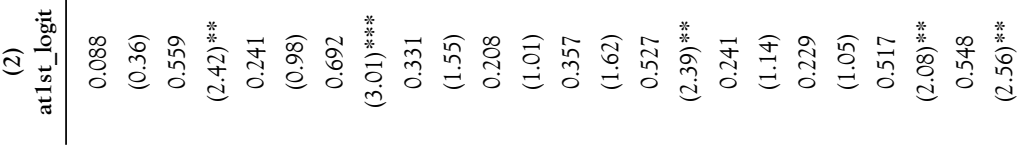

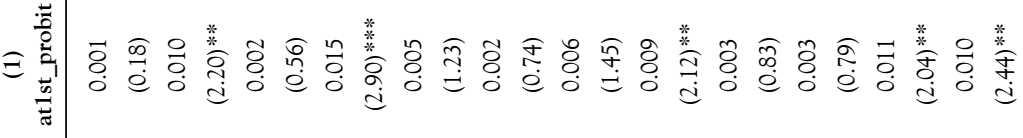

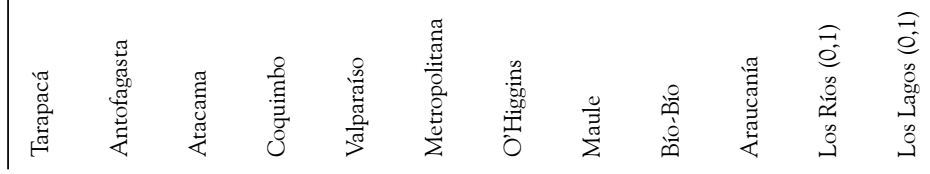




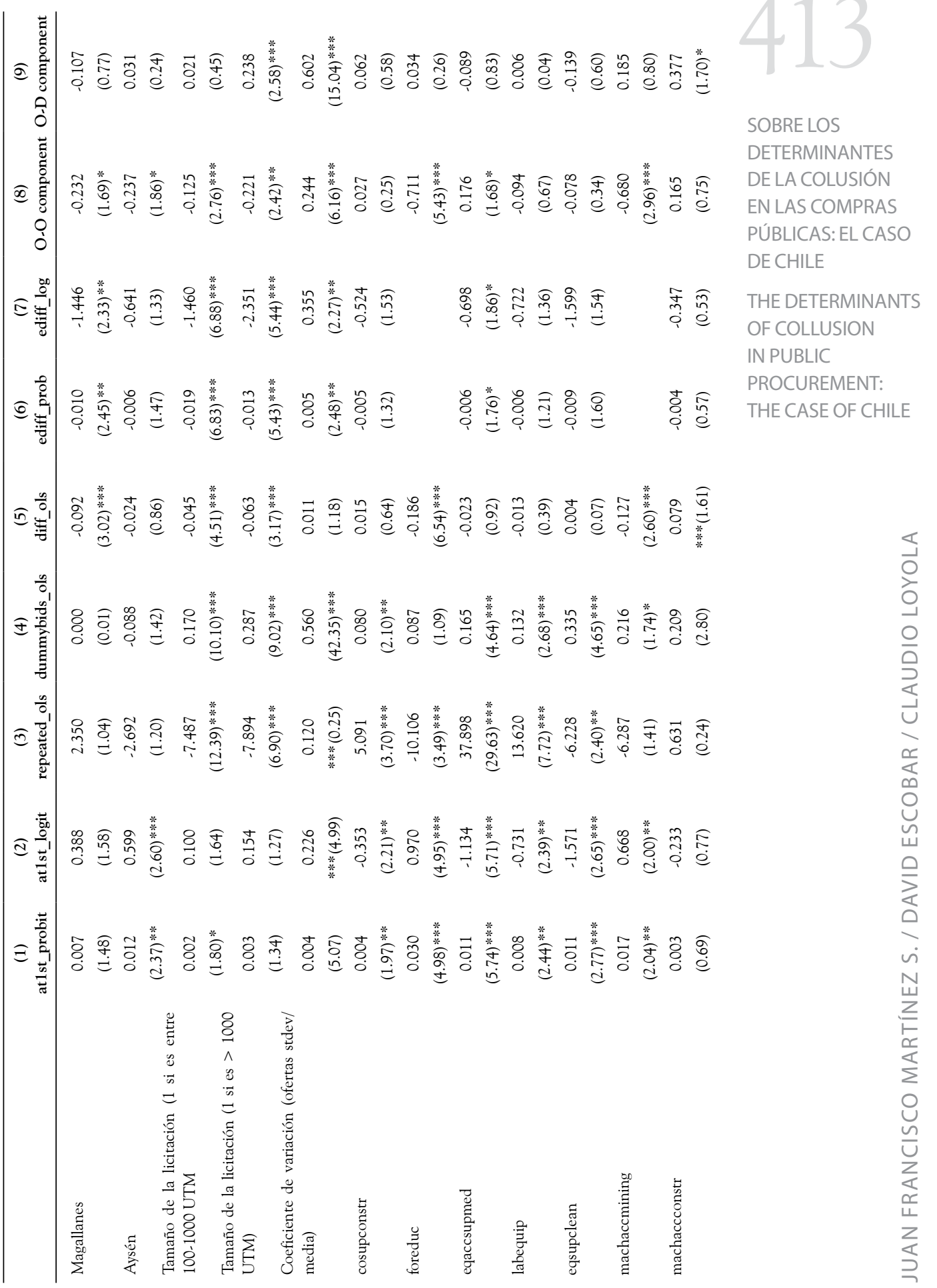




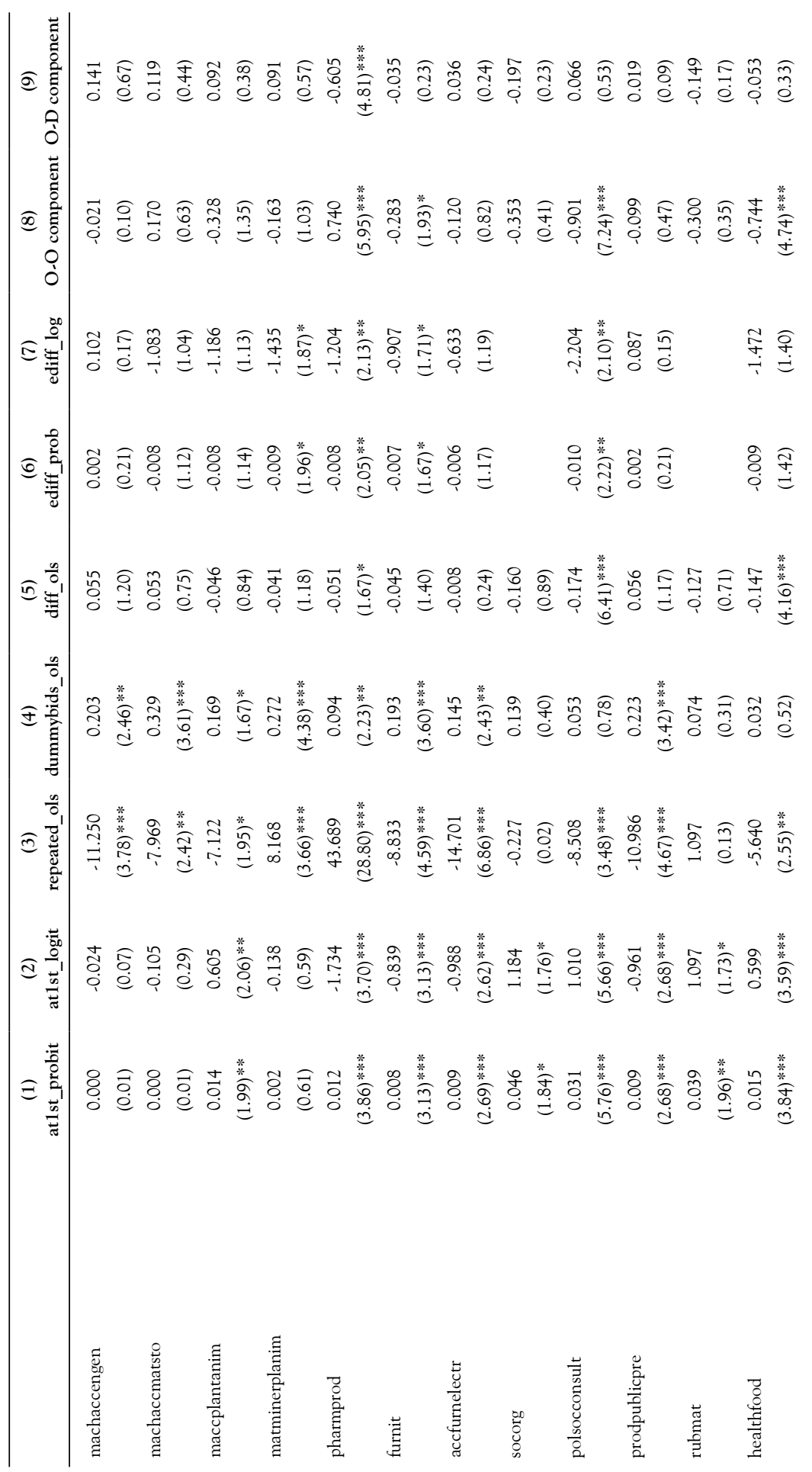




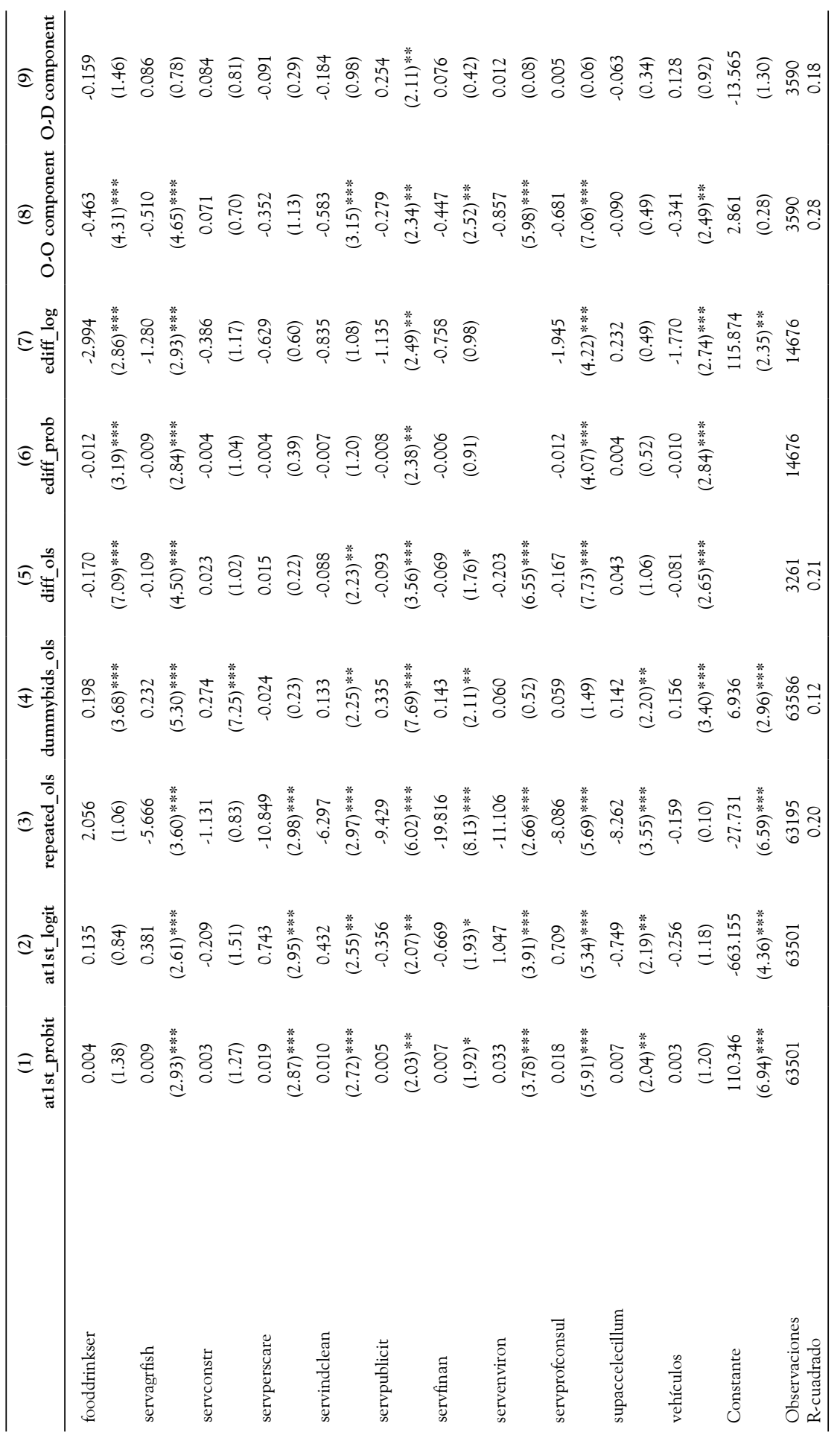

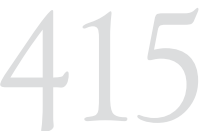

SOBRE LOS

DETERMINANTES

DE LA COLUSIÓN

EN LAS COMPRAS

PÚBLICAS: EL CASO

DE CHILE

THE DETERMINANTS

OF COLLUSION

IN PUBLIC

PROCUREMENT:

THE CASE OF CHILE 
equipos y accesorios de laboratorio; materiales de minerales, plantas o animales - una amplia variedad de materiales es usada, principalmente, para construcción liviana y tareas de reparación de las municipalidades-; productos farmacéuticos; y servicios de comida y bebestibles.

En tercer lugar, para el tipo de colusión en que existe un exceso de ofertas falsas (columna 4), los rubros afectados son demasiados para incluirlos por escrito, aunque generalmente se relacionan con la construcción y los servicios que involucran grandes inversiones - las más importantes de las cuales se pueden observar en la tabla 2-.

En cuarto lugar, para el tipo de licitación en que existen diferencias excesivas en los precios estimados y adjudicados, los rubros afectados por la colusión no están claramente definidos; es más, se podría decir que todos son afectados de igual manera.

\section{III.PRUEBAS DE ROBUSTEZ Y ANÁLISIS DE SENSIBILIDAD}

Esta sección está dedicada a verificar las especificaciones econométricas de la regresión y la robustez estadística de nuestros resultados. Primero ejecutamos regresiones sin constante pero con todas las variables ficticias para verificar si la constante agrega información o no. Este análisis se ejecuta para todas las especificaciones que tenemos. Las regresiones no varían con respecto a los resultados que obtuvimos en la sección anterior; obviamente, está aun más claro el efecto de cada variable ficticia.

En segundo lugar, ejecutamos regresiones (tabla 8b) y agregamos el coeficiente de variación de las ofertas como una variable explicativa para detectar si esta nos ayuda a explicar la colusión. Finalmente, los resultados confirman la teoría porque, a mayor variación entre ofertas - controlando por todas las demás variables-, existen mayores probabilidades de colusión. Los resultados no varían mucho con esta nueva especificación.

Por último, probamos los residuales de regresión lineal para verificar que todas las regresiones contienen ruido blanco. El análisis gráfico se muestra en el apéndice 2. Claramente, no hay preocupaciones sobre el sesgo de nuestros residuales de regresión; estos siguen la línea estándar de los supuestos de regresión.

\section{CONCLUSIONES}

Al utilizar un enfoque estándar de análisis MCO, Probit y Logit, podemos encontrar patrones de comportamiento que indican la presencia de colusión. La variabilidad explicada no es demasiado grande, debido a que es un fenómeno sutil. Sin embargo, este análisis nos permite obtener 
poderosas perspectivas para enfocarnos eficientemente en los sectores más susceptibles a la colusión.

Nuestro análisis no es completamente concluyente porque no está basado en juicios ni fallos legales; se consideran los resultados sobre la base de las anomalías en el comportamiento de las ofertas, los precios y el esquema de adjudicación. Para lograrlo, necesitaríamos una base de datos uniforme y procesada que incluya los casos en que se ha determinado legalmente un ilícito de colusión. La construcción de este tipo de base de datos sería muy importante, pero a la fecha no se ha realizado.

Hemos demostrado la robustez de nuestros resultados por medio de un sencillo conjunto de pruebas, al tomar en cuenta los supuestos econométricos y el razonamiento de las estimaciones. En un análisis posterior podríamos ejecutar una mayor diversidad de pruebas, pero suponemos que los resultados no serían muy distintos.

Claramente, la colusión puede clasificarse en dos categorías: colusión entre proveedores u oferentes $(\mathrm{O}-\mathrm{O})$, y colusión entre comprador y proveedor (O-D). Al realizar un análisis estándar de componentes principales, hemos determinado que, desde los componentes principales de las cinco variables dependientes, hay dos dimensiones principales de colusión que se relacionan con la oferta y con la relación ilícita entre oferta y demanda.

Los resultados indican que el sector productivo, la independencia del organismo comprador, el monto involucrado, la posibilidad de subcontratación, la cantidad de ofertas y el esquema de adjudicación múltiple son determinantes de la colusión. Por lo tanto, dependiendo del tipo de ilícito, existe una necesidad de concentrarse en las licitaciones de organismos compradores más independientes, con opciones de subcontratación y la posibilidad de adjudicación múltiple.

Un análisis más profundo debería incluir una estimación de datos de panel para observar la variación en el tiempo y de los grupos. Más aún, utilizando esta herramienta econométrica podríamos hacer algunas inferencias acerca de los efectos de la estacionalidad. En este sentido, uno de los análisis pendientes tiene que ver con el costo económico total de la colusión. 